\section{$\$$ Research Square}

\title{
Distributed fixed-time cooperative control of an islanded microgrid under event-triggered mechanism
}

\section{Deming Xu}

Suzhou University of Science and Technology

Ze Li ( $D$ lizeing@163.com )

Suzhou University of Science and Technology https://orcid.org/0000-0002-0354-4666

Guozeng Cui

Suzhou University of Science and Technology

Wanjun Hao

Suzhou University of Science and Technology

Fuyuan Hu

Suzhou University of Science and Technology

\section{Research Article}

Keywords: Cooperative control, Fixed-time, Eventtrigger, Islanded microgrid

Posted Date: August 17th, 2021

DOI: https://doi.org/10.21203/rs.3.rs-793535/v1

License: (c) (i) This work is licensed under a Creative Commons Attribution 4.0 International License. Read Full License 


\title{
Distributed fixed-time cooperative control of an islanded microgrid under event-triggered mechanism
}

\author{
Deming Xu • Ze Li • Guozeng Cui - Wanjun Hao - Fuyuan Hu
}

Received: date / Accepted: date

\begin{abstract}
The secondary cooperative control problem of an islanded microgrid through event-triggered mechanism is investigated in this paper. A distributed fixedtime secondary cooperative control strategy is proposed to obtain frequency and voltage magnitude secondary restoration, and also a proportional active power sharing under an undirected topology. We consider a centralized event-triggered mechanism to alleviate the communication burden and reduce the frequency of controllers update. Through this mechanism, the distributed fixed-time control protocols using frequency, voltage magnitude and active power sampling measurement values of distributed generations (DGs) only when the predefined event-triggered condition is satisfied. Compared with the conventional distributed asymptotic control protocols under period-triggered communication, the secondary control objectives of an islanded microgrid are achieved within a fixed settling time by applying the presented distributed fixed-time control approach, and the upper bound of settling time is unrelated to any initial states. Meanwhile, the presented centralized event-triggered communication method exhibits excellent performance in alleviating communication burden and promoting control efficiency. The theoretical proof is given by adopting Lyapunov method. The simulation studies are conducted to illustrate the effectiveness of the proposed control scheme.
\end{abstract}

D. Xu $\cdot$ Z. Li (凶) · G. Cui $\cdot$ W. Hao $\cdot$ F. Hu

Suzhou Institute of Intelligent City, School of Electronic and Information Engineering, Suzhou University of Science and Technology, Suzhou, 215009, China

\section{Z. Li (西)}

Suzhou Institute of Intelligent City, School of Electronic and Information Engineering, Suzhou University of Science and Technology, Suzhou, 215009, China

E-mail: lizeing@163.com
Keywords Cooperative control · Fixed-time · Eventtrigger $\cdot$ Islanded microgrid

\section{Introduction}

Recently, MG has been proposed as a key solution to cope with energy and environmental problems. MG is a small-scale power system, which consists of various distributed generations (DGs) such as wind generations, photovoltaic and fuel cells [1-3]. Note that MG is able to improve the reliability and flexibility since its two operation modes: grid-connected mode and islanded mode [4-6]. For an islanded MG, the DGs are in charge of regulating the output frequency and voltage magnitude, and also responsible for output active power sharing independently $[7,8]$. In order to ensure the reliability and stability of an islanded MG, primary control and secondary control are widely employed, where primary droop control is mainly applied to obtain the active power sharing control and guarantee the reliability of an islanded MG system [9-12]. However, the deviations of frequency and voltage magnitude are occurred due to the utilization of droop control $[13,14]$. Fortunately, the secondary control can effectively deal with the above challenges [15-18], so this paper focuses on the design of secondary control scheme for islanded MG.

Recently, the distributed cooperative control has been widely studied in islanded MG secondary control level since its advantages include reliability, flexibility and scalability [19]. The conventional distributed cooperative secondary control schemes employ asymptotic cooperative consensus control method [20-25]. The asymptotic consensus control approach could not estimate the settling time of the algorithm, and it can not meet the requirement of settling time in an realistic is- 
landed MG system. In order to obtain a faster convergence rate, the distributed finite-time control schemes were studied in [26-31]. In [26-31], the distributed finitetime control protocols were applied in the secondary control level to realize the secondary control objectives. However, the upper bound of settling time is highly dependent on the initial states of an islanded MG system. In fact, the initial states could not determine even are hard to obtain in an realistic islanded MG system. In order to solve the aforementioned issue, a series of distributed fixed-time cooperative control schemes for multi-agent system were proposed in [32-36]. Compared with the distributed finite-time control protocol, the distributed fixed-time control scheme is easier to implement in an islanded MG system since its more flexible settling time.

The conventional secondary control scheme is dependent on the continue communication, and the sampling data exchanged between DGs and controllers update is achieved in a periodic way, which could bring the excessive consumption of communication and computation resources. In order to tackle these challenges, the event-triggered control strategy has emerged and attracts a lot of attention. In reality, the event-triggered control has been proposed in multi-agent systems, and extensive works such as [37-40] promote this research continuously. These works focused on the multi-agent systems, and aim to effectively alleviate the communication and computation burden of these control systems. Consider that the islanded MG system can be modeled as a multi-agent system, which leaves the space for the application of the event-triggered control method. Inspired by the existing research works, the researchers started to extend this control approach to islanded MG system [41-46]. [41] reported a cooperative secondary voltage control scheme, which designed a auxiliary centralized event-triggered controller to reduce the communication burden. [42] proposed a distributed event-triggered cooperative control strategy, which used a fully distributed event-triggered control scheme to solve the voltage restoration problem of MGs. However, the works [41] and [42] focuses the voltage secondary control problem, and the problems of frequency and active power secondary regulation has not been touched yet. Hence, the event-triggered communication scheme of frequency and active power secondary control system was studied in [43]. In this work, the advantages of event-triggered control in reducing communication burden were validated through the comparison with traditional periodic communication scheme. The event-triggered cooperative secondary control problems includes frequency and voltage secondary restoration and active power sharing were all covered in [44] and
[45]. The distributed event-triggered scheme with cyberphysical implementation was developed in [44], and the communication burden was significantly reduced through the designed control scheme. The new distributed event-triggered restoration strategies were applied in [45] to reduce the communication burdens, and maintain the frequency and voltage magnitude restoration and the fair utilization of power sources. In [46], the centralized and distributed event-triggered control method were all got investigated in frequency and voltage secondary control strategy of islanded MGs, and the centralized approach has distinct advantage in reducing controller updates. In general, the communication burden was significantly alleviated through the eventtriggered control strategies in [41-46]. However, the aforementioned works all paid attention to first-order asymptotic cooperative control, and the new cooperative control strategy and event-triggered mechanism needed to be proposed in islanded MG system. As a result, this paper proposes a new cooperative secondary control scheme, which combines the distributed fixedtime cooperative control strategy and centralized eventtriggered control method, and aim to improve the flexibility of settling time and alleviate the communication and computation burden of an islanded MG system.

This paper investigates the secondary control problems of an islanded MG system, and a new distributed fixed-time secondary control protocol is proposed to achieve the secondary objectives, and a new centralized event-triggered mechanism is designed to alleviate the communication and computation burden. The main contributions of this paper are summarized as follows.

(1) Through utilizing the proposed distributed fixedtime secondary cooperative control algorithms, the output frequency and voltage magnitude converge to rated states while maintain the proportional active power sharing.

(2) Compared with the existing works [20-25], the proposed event-triggered fixed-time control algorithm is able to alleviate the communication burden and reduce the frequency of controllers update, and Zeno behavior can be avoided through theoretical analysis.

(3) Different from control methods in [26-31], the proposed distributed fixed-time control protocols are capable of obtaining the secondary control objectives with a fixed settling upper bound. The detailed stability analysis are provided through Lyapunov method, and the estimation method of settling time is also given.

The rest of this paper is organized as follows. The islanded MG model and some preliminaries are provided 
in section 2. The proposed control approach and stability analysis are presented in section 3 . Section 4 presents the simulation results to illustrate the effectiveness of proposed control approach. Section 5 concludes this paper.

\section{Islanded microgrid model and preliminaries}

\subsection{Graph}

This paper considers the communication topology of an islanded MG with $N$ DGs as an undirected graph $G=\{V, E, A\}$, where the $V=\{1,2, \cdots, N\}$ represents the set of nodes, and $E \subseteq V \times V$ are the set of edges. $A=\left[a_{i j}\right] \in R^{N \times N}$ is a adjacency matrix, where $a_{i i}=0$ for each DG $i$. If $\left(v_{j}, v_{i}\right) \in E$, then $a_{i j}=1$, otherwise $a_{i j}=0$. Let the Laplacian matrix as $L=D-A$, and the degree matrix is denoted as $D=\operatorname{diag}\left\{d_{1}, d_{2}, \cdots, d_{N}\right\}$, where the diagonal elemen$\mathrm{t}$ is defined as $d_{i}=\sum_{j=1, j \neq i}^{N} a_{i j}$. The pinning matrix is $B=\operatorname{diag}\left\{b_{i}\right\} \in R^{N \times N}$, and consider an islanded MG has one virtual leader node to provide reference information for DG, if DG $i$ can obtain reference values from the virtual leader node, then $b_{i}=1$, otherwise $b_{i}=0$. For an undirected graph, if DG $j$ is a neighbor of DG $i$, then $a_{i j}=a_{j i}=1$, otherwise $a_{i j}=a_{j i}=0$. Furthermore, the graph $G$ is a connected graph if there exists one path between any two nodes.

\subsection{DG model}

Fig. 1 depicts the typical control architecture of an DG in islanded MG. The control system is composed of voltage source converter (VSC), LC filter, output connector, transmission line, power, voltage and current controller, respectively. The communication network is also included in the islanded MG system.

(1) Voltage controller

The Voltage controller employs the PI control, the detailed control equations are given as follows $[1,10,26]$

$$
\begin{aligned}
\dot{\varphi}_{d i}= & v_{o d i}^{*}-v_{o d i}, \\
\dot{\varphi}_{q i}= & v_{o q i}^{*}-v_{o q i}, \\
i_{l d i}^{*}= & K_{i} i_{o d i}-\omega_{b} C_{f i} v_{o q i}+K_{P V i}\left(v_{o d i}^{*}-v_{o d i}\right) \\
& +K_{I V i} \varphi_{d i}, \\
i_{l q i}^{*}= & K_{i} i_{o q i}+\omega_{b} C_{f i} V_{o d i}+K_{P V i}\left(v_{o q i}^{*}-v_{o q i}\right) \\
& +K_{I V i} \varphi_{q i},
\end{aligned}
$$

where $v_{\text {odi }}, v_{\text {oqi }}, i_{\text {odi }}$ and $i_{\text {oqi }}$ are the direct and quadrature components of output voltage $v_{o i}$ and output cur- rent $i_{o i}$ for DG $i . K_{P V i}$ and $K_{I V i}$ are PI control parameters, $K_{i}$ is the constant control gain.

(2) Current controller

The current controller also applied PI control, the control equations are presented as follows $[1,10,26]$

$\dot{\delta}_{d i}=i_{l d i}^{*}-i_{l d i}$,

$\dot{\delta}_{q i}=i_{l q i}^{*}-i_{l q i}$,

$v_{i d i}^{*}=-\omega_{b} L_{f i} i_{l q i}+K_{P C i}\left(i_{l d i}^{*}-i_{l d i}\right)+K_{I C i} \delta_{d i}$,

$v_{i q i}^{*}=\omega_{b} L_{f i} i_{l d i}+K_{P C i}\left(i_{l q i}^{*}-i_{l q i}\right)+K_{I C i} \delta_{q i}$,

where $K_{P C i}$ and $K_{I C i}$ are the PI control parameter.

(3) Output LC filter and output connector

The dynamic model of output LC filter and output connector are given as follows $[1,10,26]$

$\dot{i}_{l d i}=-\frac{R_{f i}}{L_{f i}} i_{l d i}+\omega_{i} i_{l q i}+\frac{1}{L_{f i}} v_{i d i}-\frac{1}{L_{f i}} v_{o d i}$,

$\dot{i}_{l q i}=-\frac{R_{f i}}{L_{f i}} i_{l q i}-\omega_{i} i_{l d i}+\frac{1}{L_{f i}} v_{i q i}-\frac{1}{L_{f i}} v_{o q i}$,

$\dot{v}_{o d i}=\omega_{i} v_{o q i}+\frac{1}{C_{f i}} i_{l d i}-\frac{1}{C_{f i}} i_{o d i}$

$\dot{v}_{o q i}=-\omega_{i} v_{o d i}+\frac{1}{C_{f i}} i_{l q i}-\frac{1}{C_{f i}} i_{o q i}$

$\dot{i}_{\text {odi }}=-\frac{R_{c i}}{L_{c i}} i_{o d i}+\omega_{i} i_{o q i}+\frac{1}{L_{c i}} v_{o d i}-\frac{1}{L_{c i}} v_{b d i}$,

$\dot{i}_{o q i}=-\frac{R_{c i}}{L_{c i}} i_{o q i}-\omega_{i} i_{o d i}+\frac{1}{L_{c i}} v_{o q i}-\frac{1}{L_{c i}} v_{b q i}$.

(4) Power calculation

Through the vector control approach, the active power $P_{i}$ and reactive power $Q_{i}$ are calculated as [1]

$P_{i}=\left(v_{\text {odi }} i_{\text {odi }}+v_{\text {oqi }} i_{\text {oqi }}\right) \frac{\omega_{c}}{s+\omega_{c}}$,

$Q_{i}=\left(v_{o d i} i_{o q i}-v_{o q i} i_{o d i}\right) \frac{\omega_{c}}{s+\omega_{c}}$,

where $\omega_{c}$ represents the cut-off frequency of low-pass filters.

(5) Primary droop control

The primary droop control is commonly implemented as a local control scheme which mainly includes the frequency and voltage magnitude droop control, the detailed control laws are given as follows $[3,20,26]$

$\omega_{i}=\omega_{n i}-k_{i}^{p} P_{i}$,

$v_{i}=v_{n i}-k_{i}^{q} Q_{i}$.

where $\omega_{i}$ and $v_{i}$ represent the output frequency and voltage magnitude of DG $i$, respectively. $\omega_{n i}$ and $v_{n i}$ represent the reference values of frequency and voltage magnitude of power controller, which are provided by 


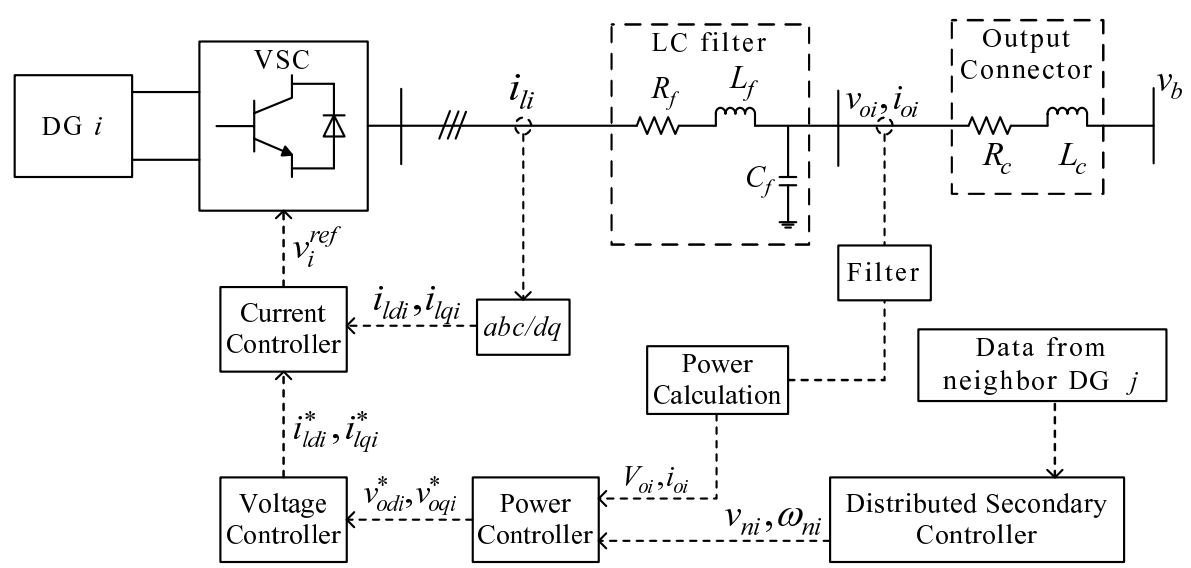

Fig. 1 The basic control structure of $i$ th DG unit

secondary controller. $k_{i}^{p}$ and $k_{i}^{q}$ are the angular frequency and voltage magnitude droop coefficient, respectively, which are set as $[1]$

$k_{i}^{p}=\frac{\omega_{\max }-\omega_{\min }}{P_{i}^{\max }}, k_{i}^{q}=\frac{v_{\max }-v_{\min }}{Q_{i}^{\max }}$,

where $\omega_{\max }$ and $\omega_{\min }$ represent the allowable maximum and minimum angular frequency, respectively. $v_{\max }$ and $v_{\min }$ represent the allowable maximum and minimum voltage magnitude, respectively. $P_{i}^{\max }$ and $Q_{i}^{\max }$ are the maximum active power and reactive power capacities of DG $i$, respectively.

The droop control inevitably causes the output frequency and voltage deviating from their rated values. As a consequence, secondary control is usually implemented in local DG to restore the output frequency and voltage magnitude. Through differentiating the droop control equation (16) and (17), we can derive

$\dot{\omega}_{i}=\dot{\omega}_{n i}-k_{i}^{p} \dot{P}_{i}=u_{i}^{\omega}$,

$\dot{v}_{i}=\dot{v}_{n i}-k_{i}^{q} \dot{Q}_{i}=u_{i}^{v}$,

where $u_{i}^{\omega}$ and $u_{i}^{v}$ represent the frequency and voltage magnitude secondary control input, respectively. Meanwhile, the active power control input is defined as $u_{i}^{p}=k_{i}^{p} \dot{P}_{i}$. Then the secondary control could also be characterized as

$\omega_{n i}=\int\left(u_{i}^{\omega}+u_{i}^{p}\right) d t$

$v_{n i}=\int\left(u_{i}^{v}+k_{i}^{q} \dot{Q}_{i}\right) d t$.

Remark 1 Compared with the MG system reference values $\omega_{\text {ref }}$ and $v_{r e f}$, the reference values of power controller $\omega_{n i}$ and $v_{n i}$ are dynamics variables, where $\omega_{n i}$ is regulated by the secondary control input $u_{i}^{\omega}$ and $u_{i}^{p}$, and $v_{n i}$ is regulated by the secondary control input $u_{i}^{v}$.

\subsection{Control objectives}

The distributed fixed-time secondary control algorithm$\mathrm{s}$ are proposed in this paper to realize the frequency and voltage magnitude restoration control, and the proportional active power sharing control with a fixed settling time. The detailed control objectives are depicted as follows

(1) Frequency and active power control

$$
\begin{aligned}
& \left\{\begin{array}{l}
\lim _{t \rightarrow T_{1}}\left|\omega_{i}(t)-\omega_{\text {ref }}\right|=0, \\
\omega_{i}(t)=\omega_{r e f}, \forall t \geq T_{1}, \forall i \in N .
\end{array}\right. \\
& \left\{\begin{array}{l}
\lim _{t \rightarrow T_{1}}\left|k_{j}^{p} P_{j}(t)-k_{i}^{p} P_{i}(t)\right|=0, \\
k_{i}^{p} P_{i}(t)=k_{j}^{p} P_{j}(t), \forall t \geq T_{1}, \forall i, j \in N .
\end{array}\right.
\end{aligned}
$$

From the definition of droop coefficient in (18), we can observe that the frequency droop coefficient is set according to the DGs' maximum active power capacity $P_{i}^{\max }$, therefore (20) is equivalent to

$$
\left\{\begin{array}{l}
\lim _{t \rightarrow T_{1}}\left|\frac{P_{i}(t)}{P_{i}^{\text {max }}}-\frac{P_{j}(t)}{P_{j}^{\text {max }}}\right|=0, \\
\frac{P_{i}(t)}{P_{i}^{\text {max }}}=\frac{P_{j}(t)}{P_{j}^{\text {max }}}, \forall t \geq T_{1}, \forall i, j \in N .
\end{array}\right.
$$

(2) Voltage magnitude restoration

$$
\left\{\begin{array}{l}
\lim _{t \rightarrow T_{2}}\left|v_{i}(t)-v_{r e f}\right|=0, \\
v_{i}(t)=v_{r e f}, \forall t \geq T_{2}, \forall i \in N .
\end{array}\right.
$$

\subsection{Preliminaries}

Assumption 1 Undirected graph $G$ is a connected graph and at least one $D G$ have reference values of the islanded $M G$ system. 
Lemma 1 [30] For $x_{1}, x_{2}, \cdots, x_{n} \geq 0$, we have

$$
\begin{aligned}
& \sum_{i=1}^{n} x_{i}^{p} \geq\left(\sum_{i=1}^{n} x_{i}\right)^{1-p}, \quad 0<p \leq 1, \\
& \sum_{i=1}^{n} x_{i}^{p} \geq n^{1-p}\left(\sum_{i=1}^{n} x_{i}\right)^{1-p}, \quad p \geq 1 .
\end{aligned}
$$

Lemma 2 [26] For an undirected and connected graph, the Laplacian matrix $L$ is a positive semi-definite matrix, and the eigenvalues of $L$ satisfy $0<\lambda_{2}(L) \leq$ $\cdots \leq \lambda_{N}(L)$. If $1^{\mathrm{T}} \mathrm{x}=0$, then $x^{\mathrm{T}} L x \geq \lambda_{2}(L) x^{\mathrm{T}} x$. Suppose that the pinning matrix has an element $b_{i} \geq 0$, then $(L+B)$ is a positive definite matrix, and define the smallest eigenvalue of $(L+B)$ as $\lambda_{1}(L+B)$, then $x^{\mathrm{T}}(L+B) x \geq \lambda_{1}(L+B) x^{\mathrm{T}} x$.

Lemma 3 [33] Consider the following nonlinear system

$\dot{x}(t)=f(x(t)), f(x(0))=0, x(t) \in R^{n}$,

where $f(): R^{n} \rightarrow R^{n}$ is a continuous vector function, and the origin is the equilibrium point of the system. If there is a continuous radially unbounded function $V$, such that the solution $V(x)$ satisfies the following inequality

$\dot{V}(x(t)) \leq-\alpha V^{p}(x(t))-\beta V^{q}(x(t))$,

where $V(x(t)) \in R^{+} \cup\{0\}, \alpha, \beta>0, p \in(0,1)$ and $q \in$ $(1, \infty)$. Fixed-time stability of the system (25) could be obtained and the settling time $T$ is described as follows

$T \leq T_{\max }:=\frac{1}{\alpha(1-p)}+\frac{1}{\beta(q-1)}$

\section{Distributed fixed-time secondary control}

In this section, the distributed fixed-time cooperative secondary control strategy is proposed. A new sampleddata-based centralized event-triggered mechanism is firstly proposed to tackle the fixed-time secondary control problems of an islanded MG. The centralized eventtriggered mechanism using the periodic sampling measurement data with a fixed period $h>0$, and the controllers also updates their control input at mutual event instants.

\subsection{Frequency and active power control}

Since the data transmission between DGs and controllers update only occur at event time, thus, the communication and computation burden of the islanded MG system is reduced by using event-triggered mechanism.
Consider the frequency droop control equation (16), the frequency varies with the output active power output, therefore we use the same event-triggered mechanism for frequency and active power secondary control, which means that the frequency and active power secondary controllers share the mutual triggering time. The specified fixed-time control rules for frequency and active power are given as follows

$$
\begin{aligned}
& u_{i}^{\omega}(t) \\
& =c_{\omega}\left[\sum_{j=1}^{N} a_{i j}\left(\omega_{j}\left(t_{k^{\prime}}^{p}\right)-\omega_{i}\left(t_{k}^{p}\right)\right)+b_{i}\left(\omega_{\text {ref }}-\omega_{i}\left(t_{k}^{p}\right)\right)\right]^{1-\frac{m}{n}} \\
& +c_{\omega}\left[\sum_{j=1}^{N} a_{i j}\left(\omega_{j}\left(t_{k^{\prime}}^{p}\right)-\omega_{i}\left(t_{k}^{p}\right)\right)+b_{i}\left(\omega_{r e f}-\omega_{i}\left(t_{k}^{p}\right)\right)\right]^{1+\frac{m}{n}} \\
& +c_{\omega}\left[\sum_{j=1}^{N} a_{i j}\left(\omega_{j}\left(t_{k^{\prime}}^{p}\right)-\omega_{i}\left(t_{k}^{p}\right)\right)+b_{i}\left(\omega_{r e f}-\omega_{i}\left(t_{k}^{p}\right)\right)\right],
\end{aligned}
$$

$$
\begin{aligned}
u_{i}^{p}(t)= & c_{p}\left[\sum_{j=1}^{N} a_{i j}\left(k_{j}^{p} P_{j}\left(t_{k^{\prime}}^{p}\right)-k_{i}^{p} P_{i}\left(t_{k}^{p}\right)\right)\right]^{1-\frac{m}{n}} \\
& +c_{p}\left[\sum_{j=1}^{N} a_{i j}\left(k_{j}^{p} P_{j}\left(t_{k^{\prime}}^{p}\right)-k_{i}^{p} P_{i}\left(t_{k}^{p}\right)\right)\right]^{1+\frac{m}{n}} \\
& +c_{p}\left[\sum_{j=1}^{N} a_{i j}\left(k_{j}^{p} P_{j}\left(t_{k^{\prime}}^{p}\right)-k_{i}^{p} P_{i}\left(t_{k}^{p}\right)\right)\right],
\end{aligned}
$$

where $c_{\omega}, c_{p}>0$ are the control gains, and $a_{i j}$ is $i j$ th element in adjacency matrix, and $m<n$ are the positive control parameters, which are an even integer and an odd integer, respectively. $k^{\prime}=: \arg \min _{\rho}\left\{t-t_{\rho} \mid t \geq\right.$ $\left.t_{\rho}, \rho \in N^{+}\right\}$. For each $t \in\left[t_{k}^{p}, t_{k+1}^{p}\right), t_{k^{\prime}}^{p}$ are the last event time of all DG. In this paper, the control law (28) and (29) for all DG are updated only at the event time $t_{0}^{p}, t_{1}^{p}, \cdots$.

A frequency deviation variable is defined as $\eta_{\omega i}(t)=$ $\omega_{i}(t)-\omega_{\text {ref }}, i \in\{1, \cdots, N\}$, and differentiate this error terms

$$
\begin{aligned}
& \dot{\eta}_{\omega i}(t)=\dot{\omega}_{i}(t)=u_{i}^{\omega}(t) \\
& =c_{\omega}\left[\sum_{j=1}^{N} a_{i j}\left(\eta_{\omega j}\left(t_{k^{\prime}}^{p}\right)-\eta_{\omega i}\left(t_{k}^{p}\right)\right)-b_{i}\left(\eta_{\omega i}\left(t_{k}^{p}\right)\right)\right]^{1-\frac{m}{n}} \\
& +c_{\omega}\left[\sum_{j=1}^{N} a_{i j}\left(\eta_{\omega j}\left(t_{k^{\prime}}^{p}\right)-\eta_{\omega i}\left(t_{k}^{p}\right)\right)-b_{i}\left(\eta_{\omega i}\left(t_{k}^{p}\right)\right)\right]^{1+\frac{m}{n}} \\
& +c_{\omega}\left[\sum_{j=1}^{N} a_{i j}\left(\eta_{\omega j}\left(t_{k^{\prime}}^{p}\right)-\eta_{\omega i}\left(t_{k}^{p}\right)\right)-b_{i}\left(\eta_{\omega i}\left(t_{k}^{p}\right)\right)\right] .
\end{aligned}
$$


Define the middle variables $\xi_{\omega i}(t), \chi_{\omega i}(t), \xi_{p i}(t)$ and $\chi_{p i}(t)$ as follows

$\xi_{\omega i}(t)=\sum_{j=1}^{N} a_{i j}\left(\eta_{\omega j}\left(t_{k^{\prime}}^{p}\right)-\eta_{\omega i}\left(t_{k}^{p}\right)\right)+b_{i}\left(\eta_{\omega i}\left(t_{k}^{p}\right)\right)$,

$\chi_{\omega i}(t)=\sum_{j=1}^{N} a_{i j}\left(\eta_{\omega j}\left(t_{k^{\prime}}^{p}\right)-\eta_{\omega i}(t)+b_{i}\left(\eta_{\omega i}(t)\right)\right.$

$\xi_{p i}(t)=\sum_{j=1}^{N} a_{i j}\left(k_{j}^{p} P_{j}\left(t_{k^{\prime}}^{p}\right)-k_{i}^{p} P_{i}\left(t_{k}^{p}\right)\right)$

$\chi_{p i}(t)=\sum_{j=1}^{N} a_{i j}\left(k_{j}^{p} P_{j}\left(t_{k^{\prime}}^{p}\right)-k_{i}^{p} P_{i}(t)\right)$,

where $t \in\left\{t_{k}^{p}, t_{k}^{p}+h, \cdots, t_{k+1}^{p}-h\right\}$, and $h$ is defined as the sampling period.

The error variable of DG $i$ is defined as follows

$$
\begin{aligned}
\delta_{i}^{p}(t)= & c_{p}\left[\xi_{p i}(t)\right]^{1-\frac{m}{n}}+c_{p}\left[\xi_{p i}(t)\right]^{1+\frac{m}{n}}+c_{p}\left[\xi_{p i}(t)\right] \\
& -c_{p}\left[\chi_{p i}(t)\right]^{1-\frac{m}{n}}-c_{p}\left[\chi_{p i}(t)\right]^{1+\frac{m}{n}}-c_{p}\left[\chi_{p i}(t)\right] .
\end{aligned}
$$

Define

$\alpha_{i}^{p}(t)=c_{p}\left|\xi_{p i}(t)\right|^{1-\frac{m}{n}}+c_{p}\left|\xi_{p i}(t)\right|^{1+\frac{m}{n}}+c_{p}\left|\xi_{p i}(t)\right|$.

In order to reduce the communication and the frequency of controllers update, the centralized eventtriggered mechanism is presented, and the centralized event-triggered function of all DG for frequency and active power secondary control have the following form:

$g_{\omega}(t)=\max \left\{\left|\delta_{i}^{p}(t)\right|-\vartheta_{1} \alpha_{i}^{p}(t)\right\}, i \in\{1, \cdots, N\}$,

where the event-triggered parameter $\vartheta_{1} \in(0,1)$.

Theorem 1 Under Assumption 1 and the distributed fixed-time secondary control laws (28) and (29), all DGs' frequency restoration and proportional active power sharing are realized in a fixed-time manner. With the proposed centralized event-triggered mechanism, the controllers are updated only when $g_{\omega}(t)>0$.

Proof Consider the following Lyapunov function candidate

$V_{1}=V_{\omega}+V_{p}=\frac{1}{2} \eta_{\omega}^{\mathrm{T}}(t)(L+B) \eta_{\omega}(t)+\frac{1}{2} P^{\mathrm{T}}(t) L P(t)$,

where $\eta_{\omega}=\left[\eta_{\omega 1}, \cdots, \eta_{\omega N}\right]^{\mathrm{T}}$ and $P=\left[k_{1}^{p} P_{1}, \cdots, k_{N}^{p} P_{N}\right]^{\mathrm{T}}$.

In light of Lemma 1, and by differentiating (38) and combine the event-triggered mechanism, we have

$\dot{V}_{1}=\eta_{\omega}^{\mathrm{T}}(t)(L+B) \dot{\eta}_{\omega}(t)+P^{\mathrm{T}}(t) L \dot{P}(t)$

$$
\begin{aligned}
& =-\sum_{i=1}^{N} \xi_{\omega i}(t) u_{i}^{\omega}(t)-\sum_{i=1}^{N} \xi_{p i}(t) u_{i}^{p}(t) \\
& =-c_{\omega} \sum_{i=1}^{N}\left[\xi_{\omega i}(t)\right]^{2-\frac{m}{n}}-c_{\omega} \sum_{i=1}^{N}\left[\xi_{\omega i}(t)\right]^{2+\frac{m}{n}} \\
& -c_{\omega} \sum_{i=1}^{N}\left[\xi_{\omega i}(t)\right]^{2}-c_{p} \sum_{i=1}^{N}\left[\xi_{p i}(t)\right]^{2-\frac{m}{n}} \\
& -c_{p} \sum_{i=1}^{N}\left[\xi_{p i}(t)\right]^{2+\frac{m}{n}}-c_{p} \sum_{i=1}^{N}\left[\xi_{p i}(t)\right]^{2} \\
& \leq \vartheta_{1} c_{\omega} \sum_{i=1}^{N}\left|\xi_{\omega i}(t)\right|^{2-\frac{m}{n}}+\vartheta_{1} c_{\omega} \sum_{i=1}^{N}\left|\xi_{\omega i}(t)\right|^{2+\frac{m}{n}} \\
& +\vartheta_{1} c_{\omega} \sum_{i=1}^{N}\left|\xi_{\omega i}(t)\right|^{2}+\vartheta_{1} c_{p} \sum_{i=1}^{N}\left|\xi_{p i}(t)\right|^{2-\frac{m}{n}} \\
& +\vartheta_{1} c_{p} \sum_{i=1}^{N}\left|\xi_{p i}(t)\right|^{2+\frac{m}{n}}+\vartheta_{1} c_{p} \sum_{i=1}^{N}\left|\xi_{p i}(t)\right|^{2} \\
& -c_{\omega} \sum_{i=1}^{N}\left[\xi_{\omega i}(t)\right]^{2-\frac{m}{n}}-c_{\omega} \sum_{i=1}^{N}\left[\xi_{\omega i}(t)\right]^{2+\frac{m}{n}} \\
& -c_{\omega} \sum_{i=1}^{N}\left[\xi_{\omega i}(t)\right]^{2}-c_{p} \sum_{i=1}^{N}\left[\xi_{p i}(t)\right]^{2-\frac{m}{n}} \\
& -c_{p} \sum_{i=1}^{N}\left[\xi_{p i}(t)\right]^{2+\frac{m}{n}}-c_{p} \sum_{i=1}^{N}\left[\xi_{p i}(t)\right]^{2} \\
& \leq \vartheta_{1} c_{\omega} \sum_{i=1}^{N}\left[\left(\xi_{\omega i}(t)\right)^{2}\right]^{\frac{2 n-m}{2 n}}+\vartheta_{1} c_{\omega} \sum_{i=1}^{N}\left[\left(\xi_{\omega i}(t)\right)^{2}\right]^{\frac{2 n+m}{2 n}} \\
& +\vartheta_{1} c_{\omega} \sum_{i=1}^{N}\left[\xi_{\omega i}(t)\right]^{2}+\vartheta_{1} c_{p} \sum_{i=1}^{N}\left[\left(\xi_{p i}(t)\right)^{2}\right]^{\frac{2 n-m}{2 n}} \\
& +\vartheta_{1} c_{p} \sum_{i=1}^{N}\left[\left(\xi_{p i}(t)\right)^{2}\right]^{\frac{2 n+m}{2 n}}+\vartheta_{1} c_{p} \sum_{i=1}^{N}\left[\xi_{p i}(t)\right]^{2} \\
& -c_{\omega} \sum_{i=1}^{N}\left[\left(\xi_{\omega i}(t)\right)^{2}\right]^{\frac{2 n-m}{2 n}}-c_{\omega} \sum_{i=1}^{N}\left[\left(\xi_{\omega i}(t)\right)^{2}\right]^{\frac{2 n+m}{2 n}} \\
& -c_{\omega} \sum_{i=1}^{N}\left[\xi_{\omega i}(t)\right]^{2}-c_{p} \sum_{i=1}^{N}\left[\left(\xi_{p i}(t)\right)^{2}\right]^{\frac{2 n-m}{2 n}} \\
& -c_{p} \sum_{i=1}^{N}\left[\left(\xi_{p i}(t)\right)^{2}\right]^{\frac{2 n+m}{2 n}}-c_{p} \sum_{i=1}^{N}\left[\xi_{p i}(t)\right]^{2} \\
& \leq-\left(1-\vartheta_{1}\right) c_{\omega} \sum_{i=1}^{N}\left[\left(\xi_{\omega i}(t)\right)^{2}\right]^{\frac{2 n-m}{2 n}} \\
& -\left(1-\vartheta_{1}\right) c_{\omega} \sum_{i=1}^{N}\left[\left(\xi_{\omega i}(t)\right)^{2}\right]^{\frac{2 n+m}{2 n}} \\
& -\left(1-\vartheta_{1}\right) c_{\omega} \sum_{i=1}^{N}\left[\xi_{\omega i}(t)\right]^{2}
\end{aligned}
$$




$$
\begin{aligned}
& -\left(1-\vartheta_{1}\right) c_{p} \sum_{i=1}^{N}\left[\left(\xi_{p i}(t)\right)^{2}\right]^{\frac{2 n-m}{2 n}} \\
& -\left(1-\vartheta_{1}\right) c_{p} \sum_{i=1}^{N}\left[\left(\xi_{p i}(t)\right)^{2}\right]^{\frac{2 n+m}{2 n}} \\
& -\left(1-\vartheta_{1}\right) c_{p} \sum_{i=1}^{N}\left[\xi_{p i}(t)\right]^{2} \\
& \leq-\left(1-\vartheta_{1}\right) c_{\omega}\left[\sum_{i=1}^{N}\left(\xi_{\omega i}(t)\right)^{2}\right]^{\frac{2 n-m}{2 n}} \\
& -\left(1-\vartheta_{1}\right) c_{\omega}\left(N^{2}+N_{0}\right)^{-\frac{m}{2 n}}\left[\sum_{i=1}^{N}\left(\xi_{\omega i}(t)\right)^{2}\right]^{\frac{2 n+m}{2 n}} \\
& -\left(1-\vartheta_{1}\right) c_{\omega} \sum_{i=1}^{N}\left[\xi_{\omega i}(t)\right]^{2} \\
& -\left(1-\vartheta_{1}\right) c_{p}\left[\sum_{i=1}^{N}\left(\xi_{p i}(t)\right)^{2}\right]^{\frac{2 n-m}{2 n}} \\
& \left.-\left(1-\vartheta_{1}\right) \vartheta_{1}\right) c_{p} \sum_{i=1}^{N}\left[\xi_{p i}(t)\right]^{2} . \\
& -\left(\sum_{i=1}^{N}\left(\xi_{p i}(t)\right)^{2}\right]^{\frac{2 n+m}{2 n}}
\end{aligned}
$$

According to the definition of $\xi_{\omega i}(t)$ and $\xi_{p i}(t)$, and by using Lemma 2, the following inequalities can be obtained

$$
\begin{aligned}
\sum_{i=1}^{N}\left(\xi_{\omega i}(t)\right)^{2} & \geq \lambda_{1}(L+B) \eta_{\omega}^{\mathrm{T}}(t)(L+B) \eta_{\omega}(t) \\
& =2 \lambda_{1}(L+B) V_{\omega}(t) \\
\sum_{i=1}^{N}\left(\xi_{p i}(t)\right)^{2} & \geq \lambda_{2}(L) P^{\mathrm{T}}(t) L P(t)=2 \lambda_{2}(L) V_{p}(t)
\end{aligned}
$$

Based on the above inequalities, (39) can be reritten as

$$
\begin{aligned}
\dot{V}_{1} & \leq-\left(1-\vartheta_{1}\right) c_{\omega}\left[2 \lambda_{1}(L+B) V_{\omega}(t)\right]^{\frac{2 n-m}{2 n}} \\
& -\left(1-\vartheta_{1}\right) c_{\omega}\left(N^{2}+N_{0}\right)^{-\frac{m}{2 n}}\left[2 \lambda_{1}(L+B) V_{\omega}(t)\right]^{\frac{2 n+m}{2 n}} \\
& -\left(1-\vartheta_{1}\right) c_{p}\left[2 \lambda_{2}(L) V_{p}(t)\right]^{\frac{2 n-m}{2 n}} \\
& -\left(1-\vartheta_{1}\right) c_{p} N^{-\frac{m}{n}}\left[2 \lambda_{2}(L) V_{p}(t)\right]^{\frac{2 n+m}{2 n}} \\
& -\left(1-\vartheta_{1}\right) c_{\omega} \sum_{i=1}^{N}\left[\varepsilon_{\omega i}(t)\right]^{2}-\left(1-\vartheta_{1}\right) c_{p} \sum_{i=1}^{N}\left[\varepsilon_{p i}(t)\right]^{2} \\
\leq & -\left(1-\vartheta_{1}\right) c_{\omega}\left[2 \lambda_{1}(L+B) V_{\omega}(t)\right]^{\frac{2 n-m}{2 n}} \\
& -\left(1-\vartheta_{1}\right) c_{\omega}\left(N^{2}+N_{0}\right)^{-\frac{m}{2 n}}\left[2 \lambda_{1}(L+B) V_{\omega}(t)\right]^{\frac{2 n+m}{2 n}} \\
& -\left(1-\vartheta_{1}\right) c_{p}\left[2 \lambda_{2}(L) V_{p}(t)\right]^{\frac{2 n-m}{2 n}} \\
& -\left(1-\vartheta_{1}\right) c_{p} N^{-\frac{m}{n}}\left[2 \lambda_{2}(L) V_{p}(t)\right]^{\frac{2 n+m}{2 n}} .
\end{aligned}
$$

\section{Let}

$$
\begin{aligned}
\rho_{1}=\min \{ & \left(1-\vartheta_{1}\right) c_{\omega}\left[2 \lambda_{1}(L+B)\right]^{\frac{2 n-m}{2 n}}, \\
& \left.\left(1-\vartheta_{1}\right) c_{p}\left[2 \lambda_{2}(L)\right]^{\frac{2 n-m}{2 n}}\right\} \\
\rho_{2}=\min \{ & \left(1-\vartheta_{1}\right) c_{\omega}\left(N^{2}+N_{0}\right)^{-\frac{m}{2 n}}\left[2 \lambda_{1}(L+B)\right]^{\frac{2 n+m}{2 n}}, \\
& \left.\left.\left(1-\vartheta_{1}\right) c_{p} N^{-\frac{m}{n}}\right)\left[2 \lambda_{2}(L)\right]^{\frac{2 n+m}{2 n}}\right\}
\end{aligned}
$$

Obviously, it follows that

$$
\begin{aligned}
\dot{V}_{1} \leq & -\rho_{1}\left(V_{\omega}\right)^{\frac{2 n-m}{2 n}}-\rho_{2}\left(V_{\omega}\right)^{\frac{2 n+m}{2 n}} \\
& -\rho_{1}\left(V_{p}\right)^{\frac{2 n-m}{2 n}}-\rho_{2}\left(V_{p}\right)^{\frac{2 n+m}{2 n}} \\
\leq & -\rho_{1}\left(V_{\omega}+V_{p}\right)^{\frac{2 n-m}{2 n}}-\rho_{2} 2^{-\frac{m}{2 n}}\left(V_{\omega}+V_{p}\right)^{\frac{2 n+m}{2 n}} \\
& -\rho_{1}\left(V_{1}\right)^{\frac{2 n-m}{2 n}}-\rho_{2} 2^{-\frac{m}{2 n}}\left(V_{1}\right)^{\frac{2 n+m}{2 n}} .
\end{aligned}
$$

According to Lemma 3, the settling time of secondary frequency and active power control is bounded as follows

$T_{1} \leq T_{1}^{\max }=\frac{2 n}{m \rho_{1}}+\frac{2 n}{m \rho_{2}} \cdot 2^{\frac{m}{2 n}}$.

Therefore, the proof of fixed-time stability for frequency and active power secondary control is completed.

Zeno behavior should be avoided in practical MG control systems, which means that the strictly positive triggering interval must be ensured. Then, Zeno behavior will not take place only if the triggering interval $t_{k+1}^{p}-t_{k}^{p}$ is greater than zero. Now, we are going to prove it through the following theorem.

Theorem 2 Under the distributed cooperative control laws (28) and (29) with the proposed event-triggering condition $g_{\omega}(t)>0$, the Zeno behavior can be avoided in the cooperative control system.

Proof Consider the consensus of active power sharing ratio and output frequency for DGs are obtained, respectively. We have $\xi_{p i}(t)=\chi_{p i}\left(t_{k}^{p}\right)=0$, then $\left|\delta_{i}^{p}(t)\right|=$ $\vartheta_{1} \alpha_{i}^{p}(t)$ can be deduced. According to the proposed event-triggering condition, we derive that the Zeno behavior will not occur when the corresponding consensus are obtained, respectively.

Next, we show that the Zeno behavior can be avoided before the corresponding consensus are achieved. According to $(45)$, we can derive that $V_{p}(t)$ is bounded, which implies that $k_{i}^{p} \dot{P}_{i}(t)$ is bounded. Assume that the upper bound of $V_{p}(t)$ and $k_{i}^{p} \dot{P}_{i}(t)$ for all DGs are $M_{1}$ and $M_{2}$, respectively. When the corresponding consensus are not achieved, there exists $i \in\{1, \cdots, N\}$ and a positive constant $\rho_{1}$ to make the following inequality holds.

$\vartheta_{1} \alpha_{i}^{p}(t) \geq \rho_{1}>0$. 
According to Lemma 2, we have

$\sum_{i=1}^{N}\left(\xi_{p i}(t)\right)^{2} \leq 2 \lambda_{N}(L) P^{\mathrm{T}}(t) L P(t) \leq 2 \lambda_{N}(L) V_{p}(t)$.

By computing the derivative of $\left|\delta_{i}^{p}(t)\right|$, we can obtain

$\left|\dot{\delta}_{i}^{p}(t)\right| \leq\left(\varpi_{1}+\varpi_{2}\right) \varpi_{3}$,

where

$\varpi_{1}=c_{p}\left(1-\frac{m}{n}\right)\left(2 \lambda_{N}(L) M_{1}\right)^{-\frac{m}{2 n}}$,

$\varpi_{2}=c_{p}\left(1+\frac{m}{n}\right)\left(2 \lambda_{N}(L) M_{1}\right)^{\frac{m}{2 n}}$,

$\varpi_{3}=\left|\sum_{j=1}^{N} a_{i j} M_{2}\right|$.

For each $t \in\left[t_{k}^{p}, t_{k+1}^{p}\right)$, we further obtain

$$
\begin{aligned}
\left|\delta_{i}^{p}(t)\right| & \leq \int_{t_{k}^{p}}^{t}\left|\dot{\delta}_{i}^{p}(s)\right| d s \\
& \leq \int_{t_{k}^{p}}^{t}\left(\varpi_{1}+\varpi_{2}\right) \varpi_{3} d s .
\end{aligned}
$$

According to the event-triggering condition $g_{\omega}(t)>$ 0 , and the inequalities (47) and (49), we derive

$t_{k+1}^{p}-t_{k}^{p} \geq \frac{\left|\delta_{i}^{p}(t)\right|}{\left(\varpi_{1}+\varpi_{2}\right) \varpi_{3}}>\frac{\rho_{1}}{\left(\varpi_{1}+\varpi_{2}\right) \varpi_{3}}>0$.

Therefore, the proposed event-triggered controller has no Zeno behavior.

\subsection{Voltage magnitude restoration}

To achieve the DGs' output voltage magnitude secondary restoration, the secondary voltage control scheme through distributed fixed-time control algorithms, and utilizes a centralized event-triggered control approach to reduce the frequency of controllers update and alleviate communication burden. The designed voltage control algorithm is presented as follows

$$
\begin{aligned}
& u_{i}^{v}(t) \\
& =c_{v}\left[\sum_{j=1}^{N} a_{i j}\left(v_{j}\left(t_{k^{\prime}}^{v}\right)-v_{i}\left(t_{k}^{v}\right)\right)+b_{i}\left(v_{r e f}-v_{i}\left(t_{k}^{v}\right)\right)\right]^{1-\frac{m}{n}} \\
& +c_{v}\left[\sum_{j=1}^{N} a_{i j}\left(v_{j}\left(t_{k^{\prime}}^{v}\right)-v_{i}\left(t_{k}^{v}\right)\right)+b_{i}\left(v_{r e f}-v_{i}\left(t_{k}^{v}\right)\right)\right]^{1+\frac{m}{n}} \\
& +c_{v}\left[\sum_{j=1}^{N} a_{i j}\left(v_{j}\left(t_{k^{\prime}}^{v}\right)-v_{i}\left(t_{k}^{v}\right)\right)+b_{i}\left(v_{r e f}-v_{i}\left(t_{k}^{v}\right)\right)\right]
\end{aligned}
$$

where $c_{v}>0$ is the control gain. Similar to control laws (28) and (29), the control law (51) is updated only at its event time $t_{0}^{v}, t_{1}^{v}, \cdots$.

Let voltage magnitude deviation as $\eta_{v i}(t)=v_{i}(t)-$ $v_{\text {ref }}, i \in\{1, \cdots, N\}$, and differentiate this error term yields

$$
\begin{aligned}
& \dot{\eta}_{v i}(t)=\dot{v}_{i}(t)=u_{i}^{v}(t) \\
& =c_{v}\left[\sum_{j=1}^{N} a_{i j}\left(\eta_{v j}\left(t_{k^{\prime}}^{v}\right)-\eta_{v i}\left(t_{k}^{v}\right)\right)+b_{i}\left(\eta_{v i}\left(t_{k}^{v}\right)\right)\right]^{1-\frac{m}{n}} \\
& +c_{v}\left[\sum_{j=1}^{N} a_{i j}\left(\eta_{v j}\left(t_{k^{\prime}}^{v}\right)-\eta_{v i}\left(t_{k}^{v}\right)\right)+b_{i}\left(\eta_{v i}\left(t_{k}^{v}\right)\right)\right]^{1+\frac{m}{n}} \\
& +c_{v}\left[\sum_{j=1}^{N} a_{i j}\left(\eta_{v j}\left(t_{k^{\prime}}^{v}\right)-\eta_{v i}\left(t_{k}^{v}\right)\right)+b_{i}\left(\eta_{v i}\left(t_{k}^{v}\right)\right)\right] .
\end{aligned}
$$

The two middle variables $\xi_{v i}(t)$ and $\chi_{v i}(t)$ are defined as follows

$\xi_{v i}(t)=\sum_{j=1}^{N} a_{i j}\left(v_{j}\left(t_{k^{\prime}}^{v}\right)-v_{i}\left(t_{k}^{v}\right)\right)+b_{i}\left(v_{r e f}-v_{i}\left(t_{k}^{v}\right)\right)$,

$\chi_{v i}(t)=\sum_{j=1}^{N} a_{i j}\left(v_{j}\left(t_{k^{\prime}}^{v}\right)-v_{i}(t)\right)+b_{i}\left(v_{r e f}-v_{i}(t)\right)$,

where $t \in\left\{t_{k}^{v}, t_{k}^{v}+h, \cdots, t_{k+1}^{v}-h\right\}$.

Define the following measurement error variable

$$
\begin{aligned}
\delta_{i}^{v}(t)= & c_{v}\left[\xi_{v i}(t)\right]^{1-\frac{m}{n}}+c_{v}\left[\xi_{v i}(t)\right]^{1+\frac{m}{n}}+c_{v}\left[\xi_{v i}(t)\right] \\
& -c_{v}\left[\chi_{v i}(t)\right]^{1-\frac{m}{n}}-c_{v}\left[\chi_{v i}(t)\right]^{1+\frac{m}{n}}-c_{v}\left[\chi_{v i}(t)\right] .
\end{aligned}
$$

Define

$\alpha_{i}^{v}(t)=c_{v}\left|\xi_{v i}(t)\right|^{1-\frac{m}{n}}+c_{v}\left|\xi_{v i}(t)\right|^{1+\frac{m}{n}}+c_{v}\left|\xi_{v i}(t)\right|$.

Then the event-triggered function of voltage magnitude is designed as

$g_{v}(t)=\max \left\{\left|\delta_{i}^{v}(t)\right|-\vartheta_{2} \alpha_{i}^{v}(t)\right\}$,

where the event-triggered parameter $\vartheta_{2} \in(0,1)$.

Theorem 3 Under Assumption 1 and the distributed fixed-time secondary control laws (51), all DGs'voltage magnitude restoration are achieved in a fixed-time manner. With the proposed centralized event-triggered mechanism, the controllers are updated only when $g_{v}(t)>0$.

Proof Consider the following Lyapunov candidate

$V_{2}=\frac{1}{2} \eta_{v}^{\mathrm{T}}(t)(L+B) \eta_{v}(t)$

where $\eta_{\omega}=\left[\eta_{\omega 1}, \cdots, \eta_{\omega N}\right]^{\mathrm{T}}$. 
By taking the time derivative of (58), the following inequality is derived as

$$
\begin{aligned}
& \dot{V}_{2}=\eta_{v}^{\mathrm{T}}(L+B) \dot{\eta}_{v}(t)=-\sum_{i=1}^{N} \xi_{v i}(t) u_{i}^{v}(t) \\
& =-c_{v} \sum_{i=1}^{N}\left[\xi_{v i}(t)\right]^{2-\frac{m}{n}}-c_{v} \sum_{i=1}^{N}\left[\xi_{v i}(t)\right]^{2+\frac{m}{n}} \\
& -c_{v} \sum_{i=1}^{N}\left[\xi_{v i}(t)\right]^{2} \\
& \leq \vartheta_{2} c_{v} \sum_{i=1}^{N}\left|\xi_{v i}(t)\right|^{2-\frac{m}{n}}+\vartheta_{2} c_{v} \sum_{i=1}^{N}\left|\xi_{v i}(t)\right|^{2+\frac{m}{n}} \\
& +\vartheta_{2} c_{v} \sum_{i=1}^{N}\left|\xi_{v i}(t)\right|^{2}-c_{v} \sum_{i=1}^{N}\left[\xi_{v i}(t)\right]^{2-\frac{m}{n}} \\
& -c_{v} \sum_{i=1}^{N}\left[\xi_{v i}(t)\right]^{2+\frac{m}{n}}-c_{v} \sum_{i=1}^{N}\left[\xi_{v i}(t)\right]^{2} \\
& \leq \vartheta_{2} c_{v} \sum_{i=1}^{N}\left[\left(\xi_{v i}(t)\right)^{2}\right]^{\frac{2 n-m}{2 n}}+\vartheta_{2} c_{v} \sum_{i=1}^{N}\left[\left(\xi_{v i}(t)\right)^{2}\right]^{\frac{2 n+m}{2 n}} \\
& +\vartheta_{2} c_{v} \sum_{i=1}^{N}\left[\xi_{v i}(t)\right]^{2}-c_{v} \sum_{i=1}^{N}\left[\left(\xi_{v i}(t)\right)^{2}\right]^{\frac{2 n-m}{2 n}} \\
& -c_{v} \sum_{i=1}^{N}\left[\left(\xi_{v i}(t)\right)^{2}\right]^{\frac{2 n+m}{2 n}}-c_{v} \sum_{i=1}^{N}\left[\xi_{v i}(t)\right]^{2} \\
& \leq-\left(1-\vartheta_{2}\right) c_{v} \sum_{i=1}^{N}\left[\left(\xi_{v i}(t)\right)^{2}\right]^{\frac{2 n-m}{2 n}} \\
& -\left(1-\vartheta_{2}\right) c_{v} \sum_{i=1}^{N}\left[\left(\xi_{v i}(t)\right)^{2}\right]^{\frac{2 n+m}{2 n}} \\
& -\left(1-\vartheta_{2}\right) c_{v} \sum_{i=1}^{N}\left[\xi_{v i}(t)\right]^{2} \\
& \leq-\left(1-\vartheta_{2}\right) c_{v}\left[\sum_{i=1}^{N}\left(\xi_{v i}(t)\right)^{2}\right]^{\frac{2 n-m}{2 n}} \\
& -\left(1-\vartheta_{2}\right) c_{v}\left(N^{2}+N_{0}\right)^{-\frac{m}{2 n}}\left[\sum_{i=1}^{N}\left(\xi_{v i}(t)\right)^{2}\right]^{\frac{2 n+m}{2 n}} \\
& -\left(1-\vartheta_{2}\right) c_{v} \sum_{i=1}^{N}\left[\xi_{v i}(t)\right]^{2} .
\end{aligned}
$$

According to the definition of $\xi_{v i}(t)$ and Lemma 2, the following inequalities is given as follows

$$
\begin{aligned}
\sum_{i=1}^{N}\left(\xi_{v i}(t)\right)^{2} & \geq \lambda_{1}(L+B) \eta_{v}^{\mathrm{T}}(t)(L+B) \eta_{v}(t) \\
& =2 \lambda_{1}(L+B) V_{2}(t) .
\end{aligned}
$$

Therefore, (59) can be formulated as

$$
\begin{aligned}
\dot{V}_{2} & \leq-\left(1-\vartheta_{2}\right) c_{v}\left[2 \lambda_{1}(L+B) V_{2}(t)\right]^{\frac{2 n-m}{2 n}} \\
& -\left(1-\vartheta_{2}\right) c_{v}\left(N^{2}+N_{0}\right)^{-\frac{m}{2 n}}\left[2 \lambda_{1}(L+B) V_{2}(t)\right]^{\frac{2 n+m}{2 n}} \\
& -\left(1-\vartheta_{2}\right) c_{v} \sum_{i=1}^{N}\left[\xi_{v i}(t)\right]^{2} \\
\leq & -\left(1-\vartheta_{2}\right) c_{v}\left[2 \lambda_{1}(L+B) V_{2}(t)\right]^{\frac{2 n-m}{2 n}} \\
& -\left(1-\vartheta_{2}\right) c_{v}\left(N^{2}+N_{0}\right)^{-\frac{m}{2 n}}\left[2 \lambda_{1}(L+B) V_{2}(t)\right]^{\frac{2 n+m}{2 n}} .
\end{aligned}
$$

Let

$$
\begin{aligned}
& \beta_{1}=\left(1-\vartheta_{2}\right) c_{v}\left[2 \lambda_{1}(L+B)\right]^{\frac{2 n-m}{2 n}}, \\
& \beta_{2}=\left(1-\vartheta_{2}\right) c_{v}\left(N^{2}+N_{0}\right)^{-\frac{m}{2 n}}\left[2 \lambda_{1}(L+B)\right]^{\frac{2 n+m}{2 n}} .
\end{aligned}
$$

Then, (61) can be rewritten as

$\dot{V}_{2} \leq-\beta_{1}\left(V_{2}\right)^{\frac{2 n-m}{2 n}}-\beta_{2}\left(V_{2}\right)^{\frac{2 n+m}{2 n}}$.

According to Lemma 3 , the settling time of voltage secondary control is bounded as follows

$T_{2} \leq T_{2}^{\max }=\frac{2 n}{m \beta_{1}}+\frac{2 n}{m \beta_{2}}$.

Therefore, the proof of fixed time stability for voltage secondary control is completed.

Theorem 4 Under distributed voltage cooperative control laws (51) with the corresponding event-triggering condition $g_{v}(t)>0$, the Zeno behavior can be avoided in voltage cooperative control system.

Proof Through applying the similar analysis process as Theorem 2, we can derive that the Zeno behavior will not occur when the consensus of DGs' voltage magnitude is achieved. Now, we are going to prove that the Zeno behavior is still not occurred before the DGs' output voltage magnitude converge to rated value.

Inspired from the proof of Theorem 2, and according to (64), we can obtain that $V_{2}(t)$ is bounded. It is easy to deduce that $\dot{v}_{i}(t)$ is also bounded. Then, we define the upper bound of $V_{2}(t)$ and $\dot{v}_{i}(t)$ as $M_{3}$ and $M_{4}$, respectively. Therefore, before the voltage magnitude consensus is obtained, there exists $i \in\{1, \cdots, N\}$ and a positive constant $\rho_{2}$ to make the following inequality holds.

$\vartheta_{2} \alpha_{i}^{v}(t)>\rho_{2}>0$.

In light of Lemma 2, we obtain

$$
\begin{aligned}
\sum_{i=1}^{N}\left(\xi_{v i}(t)\right)^{2} & \leq \lambda_{N}(L+B) \eta_{v}^{\mathrm{T}}(t)(L+B) \eta_{v}(t) \\
& \leq 2 \lambda_{N}(L+B) V_{2}(t) .
\end{aligned}
$$


For each $t \in\left[t_{k}^{p}, t_{k+1}^{p}\right)$, we can derive the following inequalities

$$
\begin{aligned}
\left|\delta_{i}^{v}(t)\right| & \leq \int_{t_{k}^{v}}^{t}\left|\dot{\delta}_{i}^{v}(s)\right| d s \\
& \leq \int_{t_{k}^{v t}}\left(\varpi_{4}+\varpi_{5}\right) \varpi_{6} d s .
\end{aligned}
$$

where

$$
\begin{aligned}
& \varpi_{4}=c_{v}\left(1-\frac{m}{n}\right)\left(2 \lambda_{N}(L+B) M_{3}\right)^{-\frac{m}{2 n}}, \\
& \varpi_{5}=c_{v}\left(1+\frac{m}{n}\right)\left(2 \lambda_{N}(L+B) M_{3}\right)^{\frac{m}{2 n}}, \\
& \varpi_{6}=\left|\sum_{j=1}^{N} a_{i j} M_{4}\right| .
\end{aligned}
$$

According to the event-triggering condition $g_{v}(t)>$ 0 , and inequalities (66) and (68), we have

$t_{k+1}^{v}-t_{k}^{v} \geq \frac{\left|\delta_{i}^{v}(t)\right|}{\left(\varpi_{4}+\varpi_{5}\right) \varpi_{6}}>\frac{\rho_{2}}{\left(\varpi_{4}+\varpi_{5}\right) \varpi_{6}}>0$.

Hence, the Zeno behavior has been excluded for the proposed event-triggered controller.

Remark 2 In this paper, the centralized event-triggered mechanisms are utilized, the local controllers using the latest sampling measurement values only at event times, and all DG share the mutual event-triggered time. This control mechanism is beneficial to improve the stability of control scheme and it is easy to implement in a realistic islanded MG system.

\section{Simulation studies}

In order to verify the correctness and effectiveness of the presented distributed fixed-time secondary control under centralized event-triggered mechanism, an islanded MG test system is built by using MATLAB/Simulink. Fig. 2 presents the basic diagram of the simulation islanded MG system and its associated communication topology. This test system includes four DGs, transmission lines and four local load units. The detailed electrical parameters are given in Table 1. The rated frequency and voltage magnitude of the simulation $\mathrm{MG}$ system are $50 \mathrm{~Hz}$ and $311 \mathrm{~V}$, respectively. The sampling period $h$ is set as $0.05 \mathrm{~ms}$. The control gains are set as $c_{\omega}=c_{p}=20$ and $c_{v}=15$, the parameters are selected as $m=2, n=3, \vartheta_{1}=0.2$ and $\vartheta_{2}=0.3$. The communication graph presented in Fig. 2 is an undirected graph, and let DG 1 as the only node obtain the systems reference values.

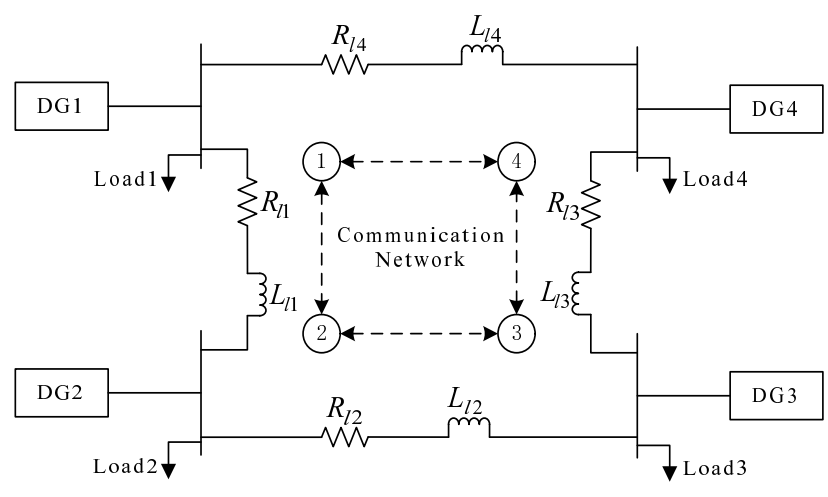

Fig. 2 Diagrams of the simulation islanded MG and its communication topology

Table 1 Parameter settling the islanded MG system

\begin{tabular}{lllll}
\hline Parameter & DG1 & DG2 & DG3 & DG4 \\
\hline$k_{i}^{p}\left(\times 10^{-5}\right)$ & 7.92 & 6.33 & 7.3 & 6.9 \\
$k_{i}^{q}\left(\times 10^{-4}\right)$ & 9.2 & 7.3 & 9.3 & 7.9 \\
$P_{i}^{\max }(\mathrm{kW})$ & 79.333 & 99.26 & 86.071 & 91.061 \\
$R_{f}(\Omega)$ & 0.15 & 0.15 & 0.15 & 0.15 \\
$L_{f}(\mathrm{mH})$ & 1.37 & 1.37 & 1.37 & 1.37 \\
$C_{f}(\mu \mathrm{F})$ & 51 & 51 & 51 & 51 \\
$R_{c}(\Omega)$ & 0.05 & 0.05 & 0.05 & 0.05 \\
$L_{c}(\mathrm{mH})$ & 0.37 & 0.37 & 0.37 & 0.37 \\
\hline & line1 & line2 & line3 & line4 \\
\hline$R_{l}(\Omega)$ & 0.79 & 0.56 & 0.25 & 0.75 \\
$L_{l}(\mathrm{mH})$ & 0.95 & 1.5 & 0.38 & 0.72 \\
\hline & load1 & load2 & load3 & load4 \\
\hline$P_{\text {load }}(\mathrm{kW})$ & 33.7 & 40.2 & 37.3 & 42.2 \\
$Q_{\text {load }}(\mathrm{kVar})$ & 12.5 & 16.2 & 12.6 & 29.6 \\
\hline
\end{tabular}

4.1 Control system performance evaluation and comparison with the method in [20]

To evaluate the control strategy's performance under load disturbances, we set an additional load suddenly connect and disconnect during the simulation process. The detailed simulation process is depicted as follows

(1) $t=0-5$ s. Only the primary control is utilized during this time period.

(2) $t=5 \mathrm{~s}$. The proposed control approach is applied.

(3) $t=10-15 \mathrm{~s}$. A constant load $L_{\text {load }}=13 \mathrm{~kW}+j 10 \mathrm{kVar}$ is connected to DG 3 .

(4) $t=15-20$ s. The load $L_{\text {load }}$ is switched off from DG 3.

The simulation results are presented in Figs. 3-6. Fig. 3 and Fig. 4 give the output frequency and voltage magnitude dynamic response, respectively. Fig. 5 presents the response of the output active power, and the response curves of DGs' output active power sharing ratio is shown in Fig. 6. Since only the primary control approach is applied during $t=0-5 \mathrm{~s}$, we can see that the DGs frequency and voltage magnitude are sta- 
bilized through applying primary control. However, the stabilized frequency and voltage magnitude all deviate from their rated values as shown in Fig. 3 and Fig. 4. As we can see from Fig. 5 and Fig. 6, the proportional active power regulation is obtained when the primary droop control is employed. Since the proposed secondary control is applied at $t=5 \mathrm{~s}$, the DGs' output frequency and voltage magnitude are restored to the rated values in a fixed-time manner, and the proportional active power sharing is also obtained within a fixed settling time. Through the simulation results, the convergence time of frequency secondary control and active power sharing control is about $0.5 \mathrm{~s}$ as presented in Fig. 3, Fig. 5 and Fig. 6. Based on (46), the settling time of the secondary frequency and active power regulation is bounded by a fixed value, which can be calculated as: $T_{1} \leq \frac{2 n}{m \rho_{1}}+\frac{2 n}{m \rho_{2}} \cdot 2^{\frac{m}{2 n}} \approx 2.6 \mathrm{~s}$. As we can see from Fig. 4, the convergence time of voltage secondary restoration is about $0.4 \mathrm{~s}$, Based on (65), the settling time of the voltage secondary control is estimated as $T_{2} \leq \frac{2 n}{m \beta_{1}}+\frac{2 n}{m \beta_{2}} \approx 3.3 \mathrm{~s}$. Therefore, the estimation method of the setting time is correct through the above simulation analysis.

To further illustrate the advantages of the proposed control approach in improving convergence rate and alleviate communication and computation burden, we compare the control method with the control scheme in [20]. In [20], the distributed asymptotical cooperative consensus control algorithm is employed to solve the secondary control problem of an islanded MG under the period-triggered mechanism. The same simulation experiment is conducted through utilizing the secondary control scheme provided in [20]. Fig. 7 and Fig. 8 present the comparison results for frequency and voltage magnitude dynamic performance in case of DG 1 , respectively, where DFTE represents the proposed control approach in this paper, and DAC denotes the control scheme in [20]. As we can observed that the proposed distributed fixed-time secondary control approach shows better convergence performance compared with the method in [20], which indicates that the proposed secondary control strategy has better robustness and convergence performance.

In order to validate the effectiveness of the presented centralized event-triggered control approach, and illustrate the advantage about reducing the controllers update. The event-triggered time for proposed secondary control at $t=5-5.01 \mathrm{~s}$ is given in Fig. 9, where ETC 1 represents the triggering mechanism for frequency and active power secondary control, ETC 2 represents the triggering mechanism for voltage magnitude secondary control. For comparison, PTC represents the periodtriggered mechanism for secondary control, which was

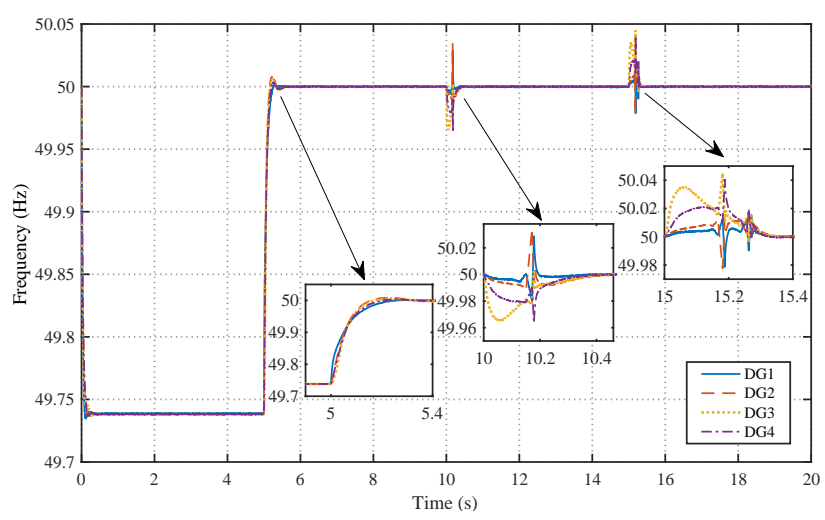

Fig. 3 DGs' output frequency

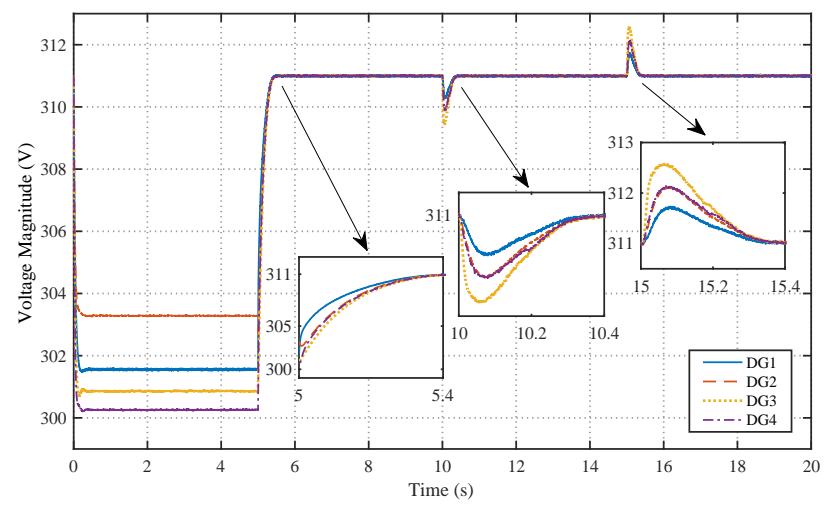

Fig. 4 DGs' output voltage magnitude

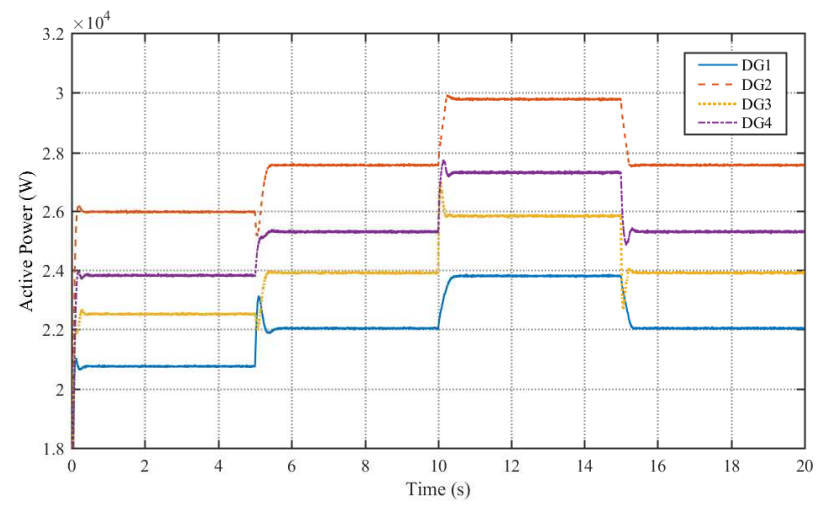

Fig. 5 DGs' output active power

utilized in [20]. Table 2 gives the total triggering number for event-triggered and period-triggered mechanism during the simulation process. Compared with periodic way, the presented event-triggered control approach exhibits a better performance in reducing the communication and computation burden. 


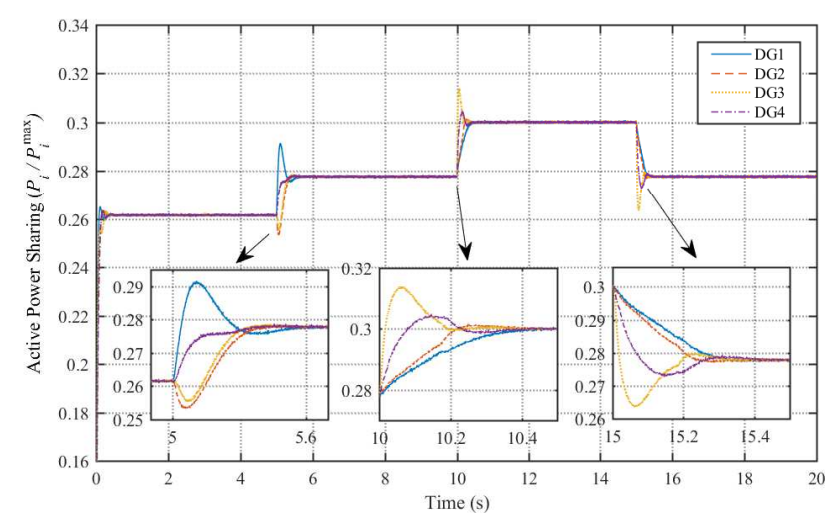

Fig. 6 DGs' output active power sharing ratio

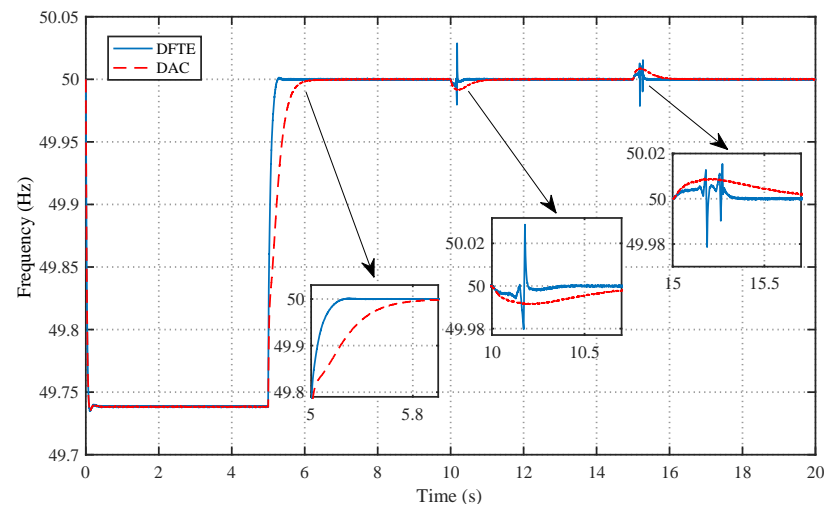

Fig. 7 The frequency convergence rate comparison with the asymptotical cooperative control method

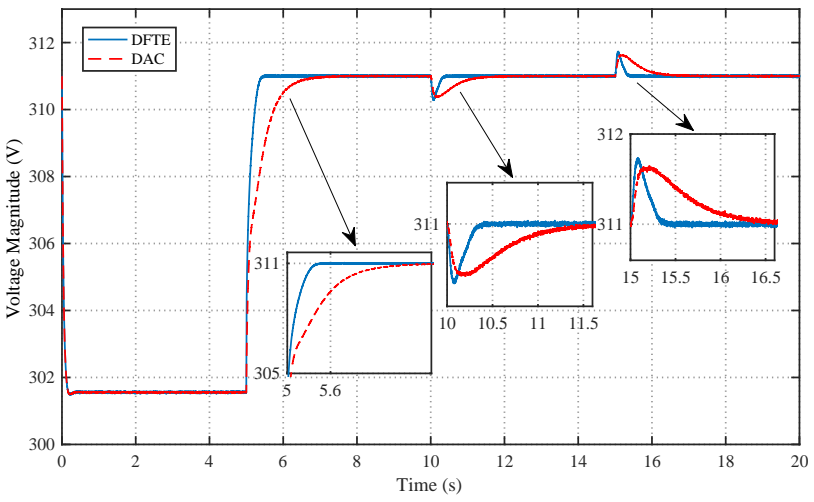

Fig. 8 The voltage magnitude convergence rate comparison with the asymptotical cooperative control method

Table 2 Comparison of update numbers under different triggering mechanism

\begin{tabular}{llccc}
\hline & DG1 & DG2 & DG3 & DG4 \\
\hline \multicolumn{5}{l}{ active power and frequency secondary control } \\
\hline ETC & 139227 & 139227 & 139227 & 139227 \\
PTC & 300000 & 300000 & 300000 & 300000 \\
\multicolumn{5}{c}{ voltage secondary control } \\
ETC & 132082 & 132082 & 132082 & 132082 \\
PTC & 300000 & 300000 & 300000 & 300000 \\
\hline
\end{tabular}
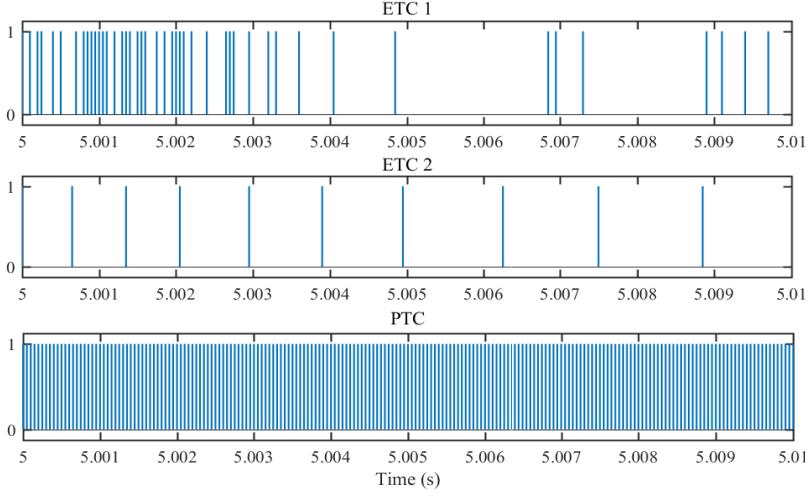

Fig. 9 Comparison of triggering time under different triggering mechanism in case of DG 1(1: Triggered. 0: Not triggered)

\subsection{Influence of communication failure}

In order to study the influence of communication link failure on control system performance, we conduct the simulation experiment, and the communication failure is arranged during the simulation process. The detailed simulation process is summarized as follows

(1) $t=2 \mathrm{~s}$. The proposed control scheme is applied.

(2) $t=5 \mathrm{~s}$. The communication link $1-4$ is cut off.

(3) $t=7 \mathrm{~s}$. The communication link $3-4$ is cut off.

(4) $t=10 \mathrm{~s}$. The load $6 \mathrm{~kW}+5 \mathrm{kVar}$ is connected to DG 3 .

(5) $t=13 \mathrm{~s}$. The communication link $1-4$ and $3-4$ is restored.

(6) $t=15 \mathrm{~s}$. The load $6 \mathrm{~kW}+5 \mathrm{kVar}$ is switched off from DG 3.

The simulation results are presented in Figs. 10-13. The proposed secondary control approach restores the frequency and voltage magnitude to rated values, and achieves the accurate active power proportional sharing when the proposed scheme is applied at 2s. Figs. 10-13 show that the link failures have barely impact on the control system performance under free of load disturbance. Since the communication link 3-4 is cut off at $t=7 \mathrm{~s}$, DG4 becomes an isolated node. Fig. 10 shows that the communication link failure has barely impact on frequency stability of the whole MG, the output frequency of all DGs are restored to the rated value after a short period of fluctuation. Fig. 12 and Fig. 13 show that the active power sharing cannot achieved when the load demand increases and the DG4 becomes an isolated node. DG1, DG2 and DG3 increase their active power output to satisfy the increased load demand. Fig.11 shows that the consensus of MG's output voltage magnitude cannot maintain since the load demand increases, the output voltage magnitude of DG4 deviating from rated value. When the communication link 


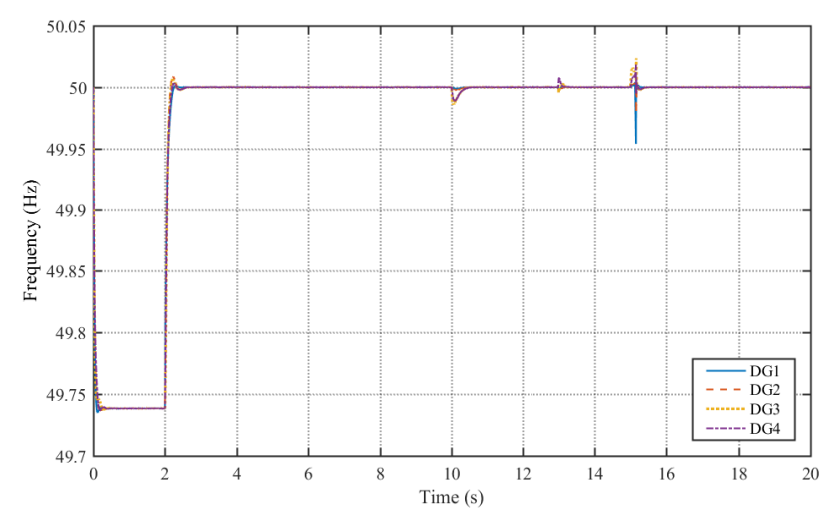

Fig. 10 DGs' output frequency in case 4.2

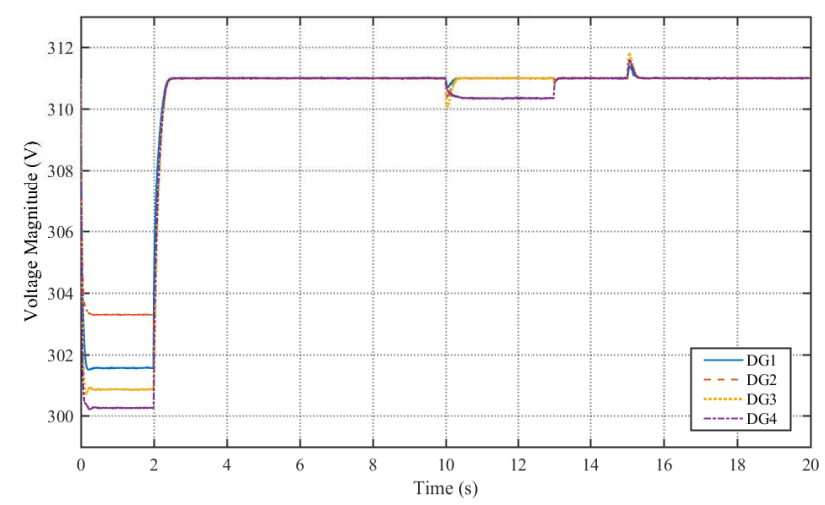

Fig. 11 DGs' output voltage magnitude in case 4.2

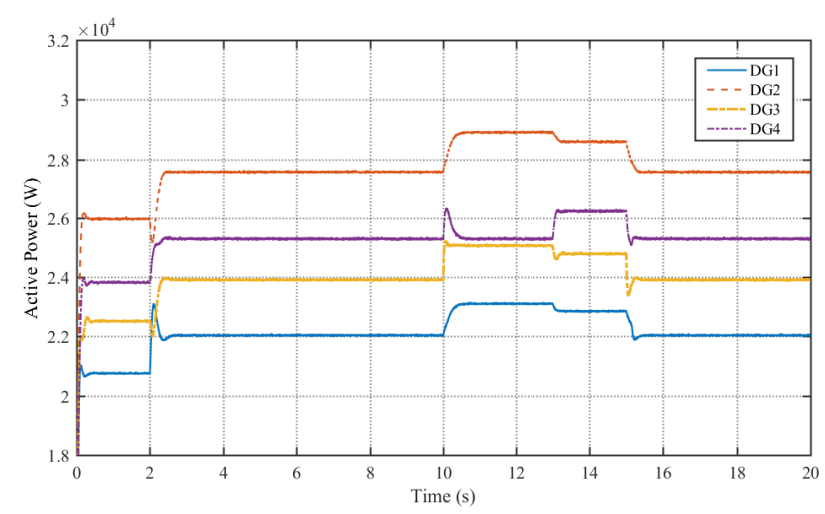

Fig. 12 DGs' output active power in case 4.2

1-4 and link 3-4 are restored at $t=13 \mathrm{~s}$, the accurate active power sharing and voltage magnitude consensus are realized with a comparatively fast speed. Therefore, we can observe that the proposed control scheme has robustness to communication link failure through the above simulation experiment.

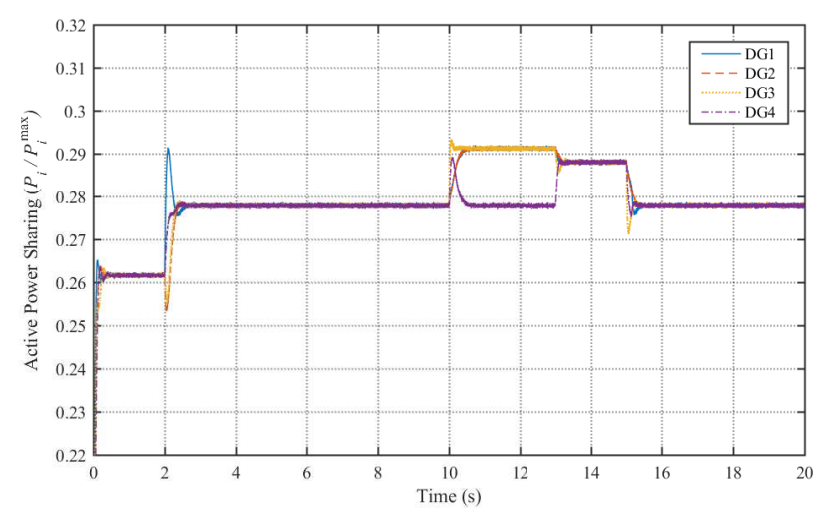

Fig. 13 DGs' output active power sharing ratio in case 4.2

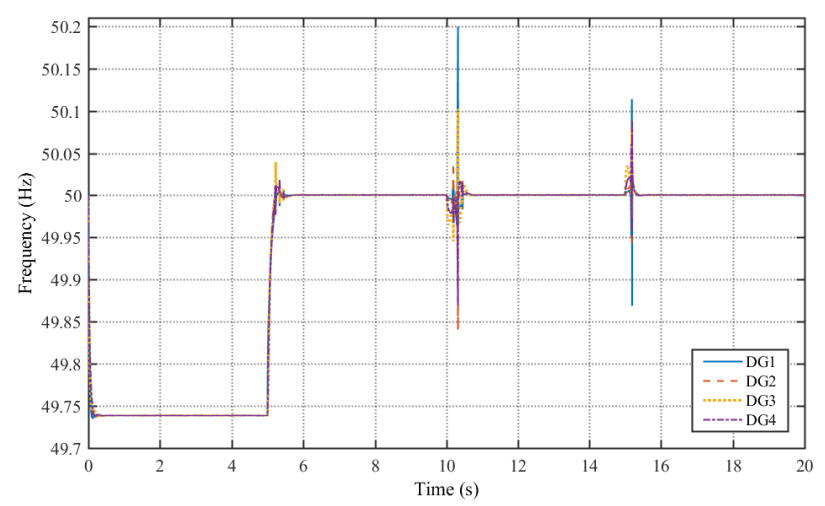

Fig. 14 DGs' output frequency in case 4.3

\subsection{Influence of communication delay}

In this case, the performance of proposed control approach is verified for delay system operation. The simulation experiment is conducted with the communication delay, the communication time delay is set as $2 \mathrm{~ms}$. The simulation parameters are set as case 4.1. The simulation results are shown as Fig. 14-17. The frequency and voltage can converge to the desired states, and accurate active power proportional sharing can also be maintain. We can see that the proposed control strategy has robustness to communication delay based on the simulation results.

4.4 Performance comparison under different control parameter $m / n$

In this case, we discuss the influence of different control parameter $m / n$. The same simulation experiments are conducted through choosing $m / n=2 / 3,2 / 5,2 / 7$, respectively. The other simulation parameters are choosing as the case 4.1. The load disturbance is also set 


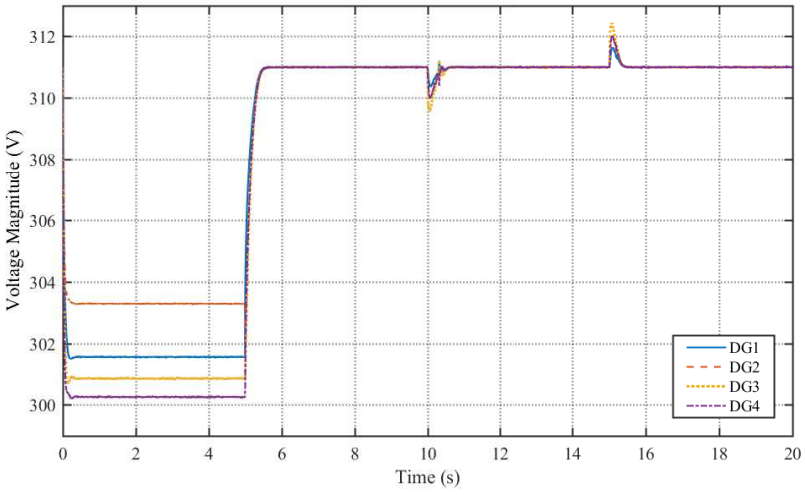

Fig. 15 DGs' output voltage magnitude in case 4.3

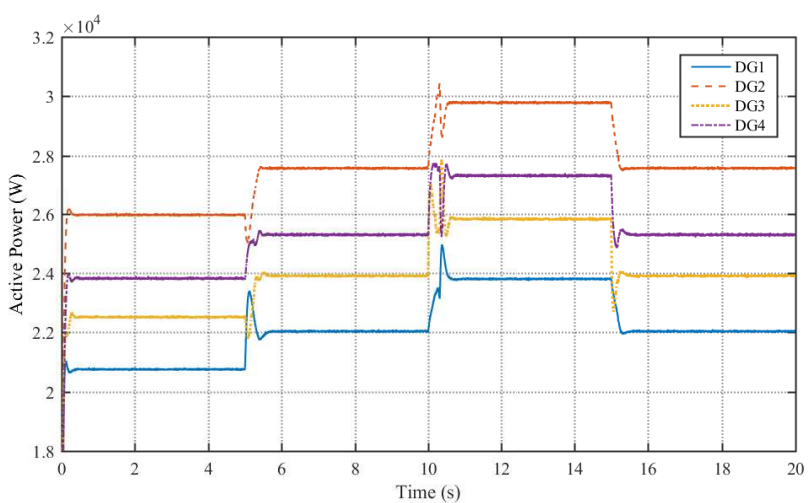

Fig. 16 DGs' output active power in case 4.3

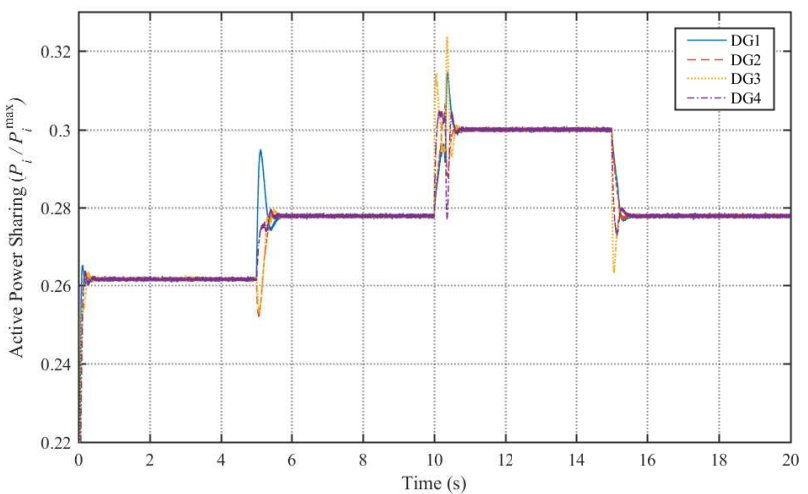

Fig. 17 DGs' output active power sharing ratio in case 4.3

during the simulation process. We compare the output frequency, output voltage magnitude and active power ratio of DG3, respectively. The comparative results are presented in Fig. 18, 19, 20. As we can observe from the simulation results, when $m / n$ is lager, the convergence speed is faster, and the convergence performance is also better.

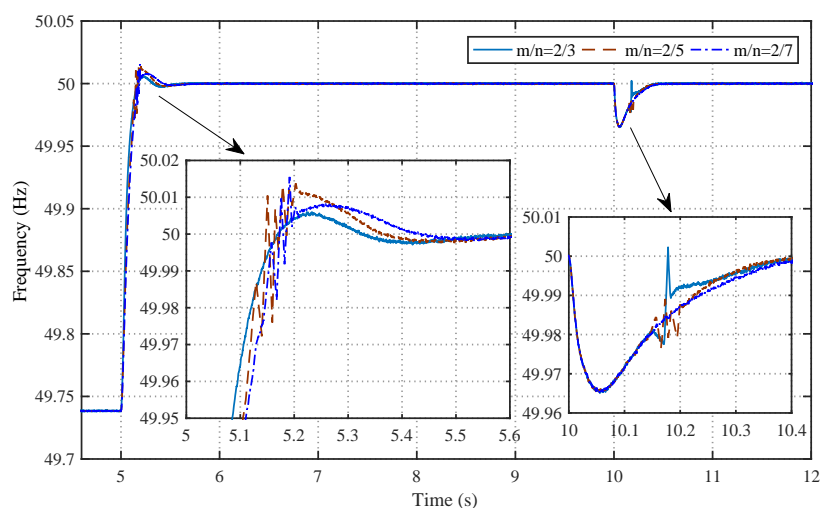

Fig. 18 The comparison of output frequency under $m / n=2 / 3,2 / 5,2 / 7$

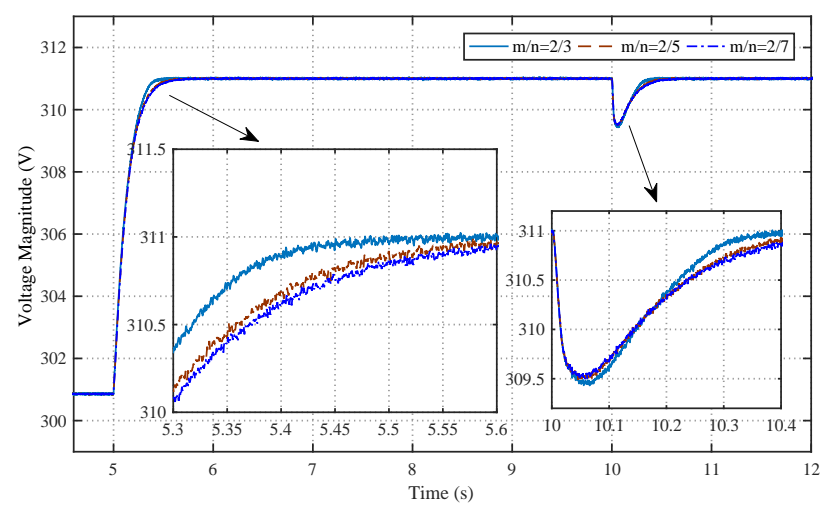

Fig. 19 The comparison of output voltage magnitude under $m / n=2 / 3,2 / 5,2 / 7$

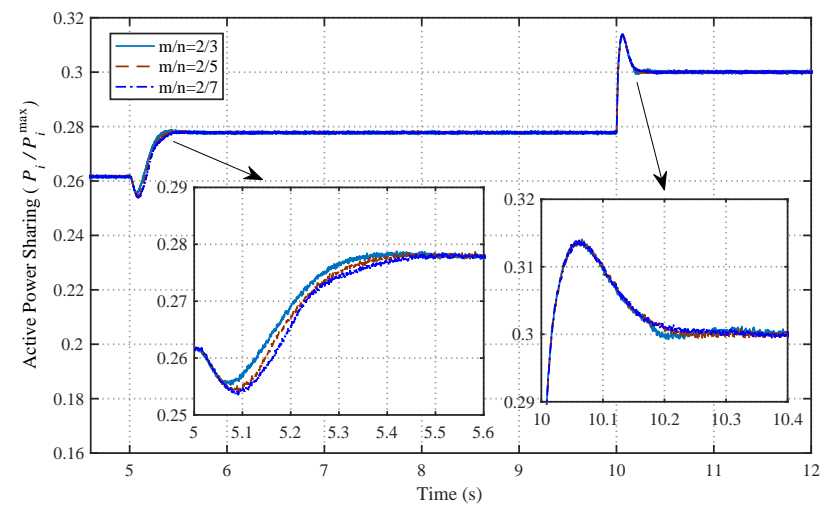

Fig. 20 The comparison of output active power sharing ratio under $m / n=2 / 3,2 / 5,2 / 7$

\section{Conclusion}

This paper has proposed the distributed fixed-time secondary control of an islanded MG under centralized event-triggered mechanism. The distributed fixed-time control algorithms were constructed for frequency and voltage magnitude restoration, and the proportional active power sharing with a fixed settling time upper 
bound. The estimation method of settling time for the proposed control algorithms have been given through adopting the Lyapunov method. A new centralized mechanism was designed to obtain the event-triggered communication, and the proposed controllers are also updated in a event-triggered way. The proposed eventtriggered mechanism is superior in terms of reducing the computation and communication burden than the traditional periodic way. Theoretical analysis and simulation results verify the effectiveness of the proposed control approach.

Funding This study was funded by the National Natural Science Foundation of China (61703059, 61873144, 61876121), and by the Science and Technology Development Plan Project of Suzhou City (SS202024).

\section{Compliance with ethical standards}

Conflict of interest The authors declare that they have no conflict of interest.

Data availability statement The authors can confirm that all data supporting the results are included in this article.

\section{References}

1. Pogaku, N., Prodanovic, M., Green, T.C.: Modeling, analysis and testing of autonomous operation of an inverterbased microgrid. IEEE Trans. Power Electron. 22(2), 613625 (2007)

2. Olivares, D.E., Mehrizi-Sani, A., Etemadi, A.H., Cañizares, C.A., Iravani, R., Kazerani, M., Hajimiragha, A.H., Gomis-Bellmunt, O., Saeedifard, M., Palma-Behnke, R., Jiménez-Estévez G.A., Hatziargyriou, N.D.: Trends in microgrid control. IEEE Trans. Smart Grid. 5(4), 1905-1919 (2014)

3. Bidram, A., Davoudi, A., Lewis, F.L.: A multiobjective distributed control framework for islanded AC microgrids. IEEE Trans. Ind. Informat. 10(3), 1785-1798 (2014)

4. Zhao, J., Dörflerb F.: Distributed control and optimization in DC microgrids. Automatica. 61, 18-26 (2015)

5. Lou, G., Gu, W., Xu, Y., Jin, W., Du, X.: Stability robustness for secondary voltage control in autonomous microgrids with consideration of communication delays. IEEE Trans. Power Syst. 33(4), 4164-4178 (2018)

6. Li, Q., Peng, C., Wang, M., Chen, M., Guerrero, J.M., Abbott, D.: Distributed secondary control and management of islanded microgrids via dynamic weights. IEEE Trans. Smart Grid. 10(2), 2196-2207 (2019)

7. Leitner, S., Yazdanian, M., Mehrizi-Sani, A., Muetze, A.: Small-signal stability analysis of an inverter-based microgrid with internal modelCbased controllers. IEEE Trans. Smart Grid. 9(5), 5393-5402 (2018)

8. Manaffam, S., Talebi, M., Jain, A.K., Behal, A.: Intelligent pinning based cooperative secondary control of distributed generators for microgrid in islanding operation mode. IEEE Trans. Power Syst. 33(2), 1364-1373 (2018)
9. Li, Z., Zang, C., Zeng, P., Yu, H., Li, S.: Fully distributed hierarchical control of parallel grid-supporting inverters in islanded AC microgrids. IEEE Trans. Ind. Informat. 14(2), 679-690 (2018)

10. Guo, Z., Li, S., Zheng, Y.: Feedback linearization based distributed model predictive control for secondary control of islanded microgrid. Asian J. Control. 22(1), 460-473 (2018)

11. Bidram, A., Poudel, B., Damodaran, L., Fierro, R., Guerrero, J.M., Fathi, M., Guerrero, J.M.: Resilient and cybersecure distributed control of inverter-based islanded microgrids. IEEE Trans. Ind. Informat. 16(6), 3881-3894 (2020)

12. Ding, L., Han, Q.-L, Ning, B., Yue, D.: Distributed resilient finite-time secondary control for heterogeneous battery energy storage systems under denial-of-service attacks. IEEE Trans. Ind. Informat. 16(7), 4909-4919 (2020)

13. Khayat, Y., Naderi, M., Shafiee, Q., Batmani, Y., Fathi, M., Guerrero, J.M., Bevrani, H.: Decentralized optimal frequency control in autonomous microgrids. IEEE Trans. Power Syst. 34(3), 2345-2353 (2019)

14. Shahab, M.A., Mozafari, B., Soleymani, S., Dehkordi, N.M., Shourkaei, H.M., Guerrero, J.M.: Distributed consensus-based fault tolerant control of islanded microgrids. IEEE Trans. Smart Grid. 11(1), 37-47 (2020)

15. Lu, X., Chen, N., Wang, Y., Qu, L., Lai, J.: Distributed impulsive control for islanded microgrids with variable communication delays. IET Control Theory Appl. 10(14), 1732-1739 (2016)

16. Ahumada, C., Cárdenas, R., Sáez, D., Guerrero, J.M.: Secondary control strategies for frequency restoration in islanded microgrids with consideration of communication delays. IEEE Trans. Smart Grid. 7(3), 1430-1441 (2016)

17. Rey, J.M., Martí, P., Velasco, M., Miret, J., Castilla, M.: Secondary switched control with no communications for islanded microgrids. IEEE Trans. Ind. Electron. 64(11), 8534-8545 (2017)

18. Guo, F., Xu, Q., Wen, C., Wang, L., Wang, P.: Distributed secondary control for power allocation and voltage restoration in islanded DC microgrids. IEEE Trans. Sustain. Ener. 9(4), 1857-1869 (2018)

19. Lu, X., Yu, X., Lai, J., Guerrero, J.M., Zhou, H.: Distributed secondary voltage and frequency control for islanded microgrids with uncertain communication links. IEEE Trans. Ind. Informat. 13(2), 448-460 (2017)

20. Bidram, A., Davoudi, A., Lewis, F.L., Qu, Z.: Secondary control of microgrids based on distributed cooperative control of multi-agent systems. IET Gener. Transm. Distrib. 7(8), 822-831 (2013)

21. Wu, X., Chen, S., Iravani, R.: A Distributed, Cooperative Frequency and Voltage Control for Microgrids. IEEE Trans. Smart Grid. 9(4), 2764-2776 (2018)

22. Dehkordi, N.M., Baghaee, H.R., Sadati, N., Guerrero, J.M.: Distributed noise-resilient secondary voltage and frequency control for islanded microgrids. IEEE Trans. Smart Grid. 10(4), 3780-3790 (2019)

23. Wu, X., Xu, Y., Wu, X., He, J., Guerrero, J.M., Liu, C.C., Schneider, K.P., Ton, D.T.: A two-layer distributed cooperative control method for islanded networked microgrid systems. IEEE Trans. Smart Grid. 11(2) 942-957 (2020)

24. Dou, C., Yue, D., Zhang, Z., Ma, K.: MAS-based distributed cooperative control for DC microgrid through switching topology communication network with timevarying delays. IEEE Syst. J. 13(1) 615-624 (2019)

25. Zhao, C., Sun, W., Wang, J., Li, Q., Mu, D., Xu, X.: Distributed cooperative secondary control for islanded microgrid with markov time-varying delays. IEEE Trans. Energy Convers. 34(3) 2235-2247 (2019) 
26. Zuo, S., Davoudi, A., Song, Y., Lewis, F.L.: Distributed finite-time voltage and frequency restoration in islanded $\mathrm{AC}$ microgrids. IEEE Trans. Ind. Informat. 63(10) 5988-5997 (2016)

27. Dehkordi, N.M, Sadati, N., Hamzeh, M.: Distributed robust finite-time secondary voltage and frequency control of islanded microgrids. IEEE Trans. Power Syst. 32(5) 36483659 (2017)

28. $\mathrm{Hu}, \mathrm{J}$, Lanzon, A.: Distributed finite-time consensus control for heterogeneous battery energy storage systems in droop-controlled microgrids. IEEE Trans. Smart Grid. 10(5) 4751-4761 (2019)

29. Xu, Y., Sun, H., Gu, W., Xu, Y., Li, Z.: Optimal distributed control for secondary frequency and voltage regulation in an islanded microgrid. IEEE Trans. Ind. Informat. 15(1) 225-235 (2019)

30. Deng, Z., Xu, Y., Sun, H., Shen, X.: Distributed, bounded and finite-time convergence secondary frequency control in an autonomous microgrid. IEEE Trans. Smart Grid. 10(3) 2776-2788 (2019)

31. Hu, Y., Wang, X., Peng, Y., Xiang, J., Wei, W.: Distributed finite-time secondary control for DC microgrid$\mathrm{S}$ with virtual impedance arrangement. IEEE Access. 7 57060-57068 (2019)

32. Anggraeni, P., Defoort, M., Djemai, M., Zuo, Z.: Control strategy for fixed-time leaderCfollower consensus for multiagent systems with chained-form dynamics. Nonlinear Dyn. 96 2693C2705 (2019)

33. Du, H., Wen, G., Wu, D., Cheng, Y., ய̈u, J.: Distributed fixed-time consensus for nonlinear heterogeneous multiagent systems. Automatica. 113108797 (2020)

34. Li, G., Wu, Y., Liu, X.: Adaptive fixed-time consensus tracking control method for second-order multi-agent systems with disturbances. J. Frankl. Inst. 357(3) 1516-1531 (2020)

35. Wang, Z., Shan, J.: Fixed-time consensus for uncertain multi-agent systems with actuator faults. J. Frankl. Inst. 357(2) 1199-1220 (2020)

36. Yu, Z., Yu, S., Jiang, H., Mei, X.: Distributed fixed-time optimization for multi-agent systems over a directed network. Nonlinear Dyn. 103 775-789 (2021)

37. Luo, L., Mi, W., Zhong, S.: Adaptive consensus control of fractional multi-agent systems by distributed eventtriggered strategy. Nonlinear Dyn. 100 1327-1341 (2020)

38. Hu, A., Park, J.H., Hu, M.: Consensus of nonlinear multiagent systems with intermittent dynamic event-triggered protocols. Nonlinear Dyn. 104 1299-1313 (2021)

39. Zhang, J., Dai, J., Zhang, H., Sun, S.: Cooperative output regulation of heterogeneous linear multi-agent system$\mathrm{s}$ based on the event-triggered distributed control under switching topologies. Appl. Math. Comput. 390125611 (2021)

40. Mu, R., Wei, A., Li, H., Yue, L.: Leader-following consensus for multi-agent systems with actuator faults via adaptive event-triggered control. J. Frank. Inst. 358(2) 13271349 (2021)

41. Chen, M., Xiao, X.: Secondary voltage control in islanded microgrids using event-triggered control. IET Gener. Transm. Distrib. 12(8) 1872-1878 (2018)

42. Xie, Y., Lin, Z.: Distributed event-triggered secondary voltage control for microgrids with time delay. IEEE Trans. Syst. Man. Cybern. Syst. 49(8) 1582-1591 (2019)

43. Ding, L., Han, Q.-L, Zhang, X.-M.: Distributed secondary control for active power sharing and frequency regulation in islanded microgrids using an event-triggered communication mechanism. IEEE Trans. Ind. Informat. 15(7) 39103922 (2019)
44. Wang, Y., Nguyen, T.L., Xu, Y., Li, Z., Tran, Q.-T., Caire, R.: Cyber-physical design and implementation of distributed event-triggered secondary control in islanded microgrids. IEEE Trans. Ind. Informat. 55(6) 5631-5642 (2019)

45. Weng, S., Yue, D., Dou, C., Shi, J., Huang, C.: Distributed event-triggered cooperative control for frequency and voltage stability and power sharing in isolated inverterbased microgrid. IEEE Trans. Cybern. 49(4) 1427-1439 (2019)

46. Qian, T., Liu, Y., Zhang, W., Tang, W., Shahidehpour, M.: Event-triggered updating method in centralized and distributed secondary controls for islanded microgrid restoration. IEEE Trans. Smart Grid. 11(2) 1387-1395 (2020) 